University of Wollongong

Research Online

Faculty of Engineering and Information

Faculty of Engineering and Information

Sciences - Papers: Part A

Sciences

$1-1-2014$

\title{
A new empirical model for shear strength of reinforced concrete beam - column connections
}

Tung Tran

University of Wollongong, tmt954@uowmail.edu.au

Muhammad N. S Hadi

University of Wollongong, mhadi@uow.edu.au

Thong M. Pham

University of Wollongong, tmp@uow.edu.au

Follow this and additional works at: https://ro.uow.edu.au/eispapers

Part of the Engineering Commons, and the Science and Technology Studies Commons

Research Online is the open access institutional repository for the University of Wollongong. For further information contact the UOW Library: research-pubs@uow.edu.au 


\title{
A new empirical model for shear strength of reinforced concrete beam - column connections
}

\author{
Abstract \\ A new empirical model to estimate the joint shear strength of both exterior and interior beam-column \\ connections is proposed. In the model, four parameters that have the most influence on joint shear \\ strength are considered. Among these four, a new parameter is introduced to consider the bond condition \\ and the possibility of beam bars transferring joint shear force into the columns. Consideration of this \\ parameter in the model significantly improves the accuracy of the predicted joint shear strength. To \\ calibrate the model, a large database of 98 reinforced concrete (RC) exterior and 73 RC interior beam- \\ column connections displaying joint failure mode was compiled from the literature. A parametric study \\ was also carried out to evaluate the dependence of the predicted to tested joint shear strength ratio on \\ the four influence parameters using the database. The proposed model showed superior performance \\ over existing models. Moreover, comparisons of the predicted joint shear strength with experimental \\ results and with four existing models showed the accuracy of the proposed model.
}

\section{Keywords}

empirical, beam, concrete, reinforced, connections, strength, column, shear, model

\section{Disciplines}

Engineering | Science and Technology Studies

\section{Publication Details}

Tran, T. M., Hadi, M. N. S. \& Pham, T. M. (2014). A new empirical model for shear strength of reinforced concrete beam - column connections. Magazine of Concrete Research, 66 (10), 514-530. 


\section{A new empirical model for shear strength of reinforced concrete beam-column connections}

Tung M. Tran

PhD Scholar, School of Civil, Mining and Environmental Engineering,

University of Wollongong, Wollongong, NSW, Australia; Lecturer,

Department of Civil Engineering, Ton Duc Thang University, Ho Chi Minh, Vietnam

Muhammad N. S. Hadi

Associate Professor, School of Civil, Mining and Environmental

Engineering, University of Wollongong, Wollongong, NSW, Australia

\section{Thong M. Pham}

PhD Candidate, School of Civil, Mining and Environmental Engineering, University of Wollongong, Wollongong, NSW, Australia; Lecturer, Faculty of Civil Engineering, HCMC University of Technology, Ho Chi Minh, Vietnam

A new empirical model to estimate the joint shear strength of both exterior and interior beam-column connections is proposed. In the model, four parameters that have the most influence on joint shear strength are considered. Among these four, a new parameter is introduced to consider the bond condition and the possibility of beam bars transferring joint shear force into the columns. Consideration of this parameter in the model significantly improves the accuracy of the predicted joint shear strength. To calibrate the model, a large database of 98 reinforced concrete (RC) exterior and $73 \mathrm{RC}$ interior beam-column connections displaying joint failure mode was compiled from the literature. A parametric study was also carried out to evaluate the dependence of the predicted to tested joint shear strength ratio on the four influence parameters using the database. The proposed model showed superior performance over existing models. Moreover, comparisons of the predicted joint shear strength with experimental results and with four existing models showed the accuracy of the proposed model.

\section{Notation}

$A_{\text {jh }} \quad$ effective joint area

$A_{\mathrm{sb}} \quad$ greater area of top or bottom beam bars

$A_{\text {sjh }}, A_{\text {sjv }}$ total area of horizontal and vertical shear reinforcement respectively

$A_{\text {str }} \quad$ effective area of the diagonal strut

$a_{i} \quad$ influence coefficients

BI beam reinforcement index

$b_{\mathrm{b}}, b_{\mathrm{c}} \quad$ width of the beam and the column sections respectively

$b_{\mathrm{j}} \quad$ effective joint width

$c \quad$ intercept value

$d_{\mathrm{sb}} \quad$ average diameter of beam tensile reinforcement

$f_{\mathrm{c}}^{\prime} \quad$ cylinder compressive strength of concrete

$f_{\text {jhy }}, f_{\text {jvy }}$ yield strength of horizontal and vertical shear reinforcement respectively

$h_{\mathrm{b}} \quad$ height of beam cross-section

$h_{\mathrm{c}} \quad$ height of column cross-section

JI joint transverse reinforcement index

$K \quad$ factor of horizontal and vertical joint shear reinforcement

$k \quad$ total number of collected experimental results

$k_{1}, k_{2}$, joint shear factors

$N \quad$ column axial load

$n \quad$ number of influence parameters
$V_{\text {ch }}$

$V_{\text {jh }}$

$V_{\text {jh,model }}$

$V_{\text {jh,test }}$

$V_{\text {sh }}$

$x_{i}$

$\alpha$

$\alpha_{\mathrm{NZS}}$

$\alpha_{\mathrm{t}}$

$\beta \quad$ factor equal to $1 \cdot 0$ and $0 \cdot 8$ for interior and exterior

joints respectively

$\beta_{\mathrm{t}} \quad$ factor describing out-of-plane geometry

$\gamma_{\mathrm{ACI}} \quad$ joint shear factor in ACI 352R-02 (ACI, 2002)

$\gamma_{1}, \gamma_{2} \quad \gamma_{1}=0.81, \gamma_{2}=0.14$ for interior joints; $\gamma_{1}=0.0 .34$, $\gamma_{2}=0.22$ for exterior joints

$\zeta \quad$ softening coefficient

$\eta_{\mathrm{t}} \quad$ parameter to account for the influence of beam eccentricity

$\theta \quad$ inclination of the diagonal compression strut from the beam longitudinal direction

$\rho_{\mathrm{sb}} \quad$ beam reinforcement ratio 


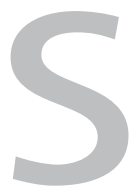

$\sigma_{\mathrm{y}}$

column axial stress

$\chi_{\mathrm{b}} \quad$ beam bar index

$\chi_{\mathrm{j}} \quad$ normalised joint shear reinforcement

$\chi_{\text {jh }} \quad$ normalised horizontal joint shear reinforcement

$\chi_{\mathrm{jv}} \quad$ normalised vertical joint shear reinforcement

\section{Introduction}

Beam-column connections are known to be one of the most critical regions of reinforced concrete (RC) structures as failure of the connections under seismic loading often leads to partial or total collapse of the whole structure. To improve the safety of RC structures under seismic load, designers have to carefully consider the shear strength and the ductility performance of beam-column connections to ensure that brittle shear failure at the joint region is avoided.

To understand the behaviour of beam-column connections, numerous experimental and analytical studies have been conducted since the mid-1960s. The first studies on beam-column connections were carried out by Hanson and Connor (1967). These were then developed by Zhang and Jirsa (1982), Sarsam and Phillips (1985), Pantazopoulou and Bonacci (1992), Hwang and Lee (1999). The latest studies on beam-column connections include those of Kim et al. (2009), Choi and Kim (2011), Joyklad et al. (2012), Kim and Yu (2012), Najafian et al. (2013) and Patel et al. (2013). Although many efforts have been made, the research community has not yet understood the full behaviour of $\mathrm{RC}$ beam-column connections because of the large variations in both geometry and distribution of forces that occur in a relatively small volume at the joint region (Pantazopoulou and Bonacci, 1992). This point of view is evident when considering the inconsistencies in existing design standards for predicting the shear strength of RC beam-column connections (ACI, 2008; AIJ, 1999; BSI, 2004; SANZ, 1995).

In general, to carry the joint shear forces, resisting mechanisms, including diagonal struts and/or trusses, are developed. The truss mechanism is justified when the bond between the concrete and the beam and column reinforcement is perfect. In this case, the joint core is considered as a uniform plane zone subjected to shear stress and joint shear reinforcement is thus required to prevent shear failure of the joint core by diagonal principal tension stress. The diagonal strut mechanism is developed by the internal forces generated in the concrete and thus joint shear reinforcement is required to provide sufficient confinement for improving the compressive strength of the concrete diagonal strut(s). Different from the truss mechanism, in the diagonal strut mechanism, the bond between the beam bars and the concrete is allowed to deteriorate. Based on the above resisting mechanisms, numerous models have been developed for predicting the shear strength of beam-column connections. These theoretical models were developed based on either the average stress approach with compatibility of strains and stress equilibrium, or the strut-and-tie approach. In addition, because the mechanism of the joint is complicated and depends on many parameters, empirical and semi-empirical models have also been developed based on experimental data.

Most of the theoretical and empirical models currently available in the literature were summarised by Lima et al. (2012). Their summary showed that, in total, 11 parameters (including geometric and mechanical parameters) were taken into account by the available capacity shear strength models. Interestingly, these 11 parameters did not include the number and diameter of beam longitudinal bars, although these factors are the most important parameters that control the mechanism of bond forces transferring from the beam reinforcement to the concrete at the joint area. Analysis of a large experimental database of 171 beam-column connections introduced in the following section showed that, in addition to parameters such as concrete compressive strength, joint shear reinforcement and column axial stress, the diameter and number of beam bars are important factors for the shear strength of a beam-column connection. Therefore, in this work, the number and diameter of beam bars were considered in order to develop an empirical shear strength model for predicting the shear strength of beam-column connections. Development of the model was based on regression analysis using a large database collected from published works. The superiority of the proposed model was evaluated by comparing the predicted joint shear strengths with 171 test results from the literature and with four existing analytical models.

\section{Experimental database}

A database of 171 experimental RC beam-column connections (98 exterior and 73 interior) was compiled from the published literature (Alva et al., 2007; Antonopoulos and Triantafillou, 2003; Attaalla, 2004; Au et al., 2005; Chalioris et al., 2008; Chun et al., 2009; Chutarat and Aboutaha, 2003; Clyde et al., 2000; Dhakal et al., 2005; Durrani and Wight, 1985; Ehsani and Alameddine, 1991; Ehsani and Wight, 1985; Ehsani et al., 1987; El-Amoury and Ghobarah, 2002; Fisher and Sezen, 2011; Ghobarah and El-Amoury, 2005; Ghobarah and Said, 2001, 2002; Hwang et al., 2004, 2005; Ishibashi, 1993; Kaku and Asakusa, 1991; Karayannis and Sirkelis, 2005, 2008; Karayannis et al., 2008; Kitayama et al., 1991; Kuang and Wong, 2006; Le-Trung et al., 2010; Lee et al., 2010; Leon, 1990; Liu, 2006; Lu et al., 2012; Megget, 1974; Meinheit and Jirsa, 1977; Morita et al., 1999; Murty et al., 2003; Noguchi and Kashiwazaki, 1992; Noguchi and Kurusu, 1988; Oka and Shiohara, 1992; Otani et al., 1984; Pantelides et al., 2002; Park and Paulay, 1974; Shrestha et al., 2009; Supaviriyakit and Pimanmas, 2008; Tsonos, 2007; Tsonos et al., 1992; Vatani-Oskouei, 2010; Wang and Hsu, 2009; Wong and Kuang, 2008).

All of the specimens were subjected to quasi-static cyclic lateral loading and were at least one-third scale. The final failure modes of the collected specimens were either joint shear or joint shear with yielding of beam reinforcement. All of the specimens had no out-of-plane members (slabs and/or transverse beams) and no eccentricity between beams and columns. Specimens that failed 


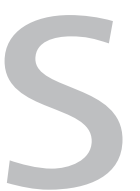

in terms of weak column-strong beam were excluded from the collected data because, in these cases, the column flexural capacity is relatively low and thus failure of the column caused by flexural moment can occur before the shear strength of the connection is reached. For interior connections, the column and beam reinforcement continuously passed through the joint panel. For exterior joints, only those specimens with conventional reinforcement anchorage were included (i.e. the longitudinal bars of the beams were anchored by hooks towards the core of the exterior joints). In the collected database, the test shear forces, $V_{\mathrm{jh}, \text { test }}$, were either collected from the reported values or derived using the maximum applied load measured from the test. In this calculation, the moment arm of the beam cross-section was assumed to be $80 \%$ of the total beam height $h_{\mathrm{b}}$.

The collected database covered a broad range of various parameters, including joint reinforcement ratio, reinforcement yielding stress, concrete strength, column axial average stresses and beam height to column height ratio $\left(h_{\mathrm{b}} / h_{\mathrm{c}}\right)$, as summarised in Table 1 for exterior joints and Table 2 for interior joints. Of the exterior connections, 46 specimens failed through joint shear and 52 specimens failed in joint shear with yielding of beam reinforcement; the corresponding numbers for the interior connections were 41 and 32 respectively.

The definitions and the ranges of the main parameters in the collected database are summarised in Table 3 , in which $N$ is the column axial load, $A_{\text {sjh }}$ and $f_{\text {jhy }}$ are the area and yield strength of the horizontal joint shear reinforcement placed between the top and the bottom beam reinforcement respectively, $A_{\mathrm{sjv}}$ and $f_{\mathrm{jvy}}$ are the area and yield strength of the intermediate vertical reinforcement passing through the joint respectively, $b_{\mathrm{j}}=\left(b_{\mathrm{c}}+b_{\mathrm{b}}\right) / 2$ is the effective joint width $\left(b_{\mathrm{c}}\right.$ and $b_{\mathrm{b}}$ are the section widths of the beam and the column respectively), $n_{\mathrm{b}}$ is the maximum number of the top and the bottom beam bars and $d_{\mathrm{sb}}$ is the corresponding average beam bar diameter.

A new parameter $\chi_{\mathrm{b}}$, referred to as the beam bar index, is proposed. From its definition in Table 3 , it can be seen that $\chi_{\mathrm{b}}$ is a function of the number of beam bars $n_{\mathrm{b}}$ and their average diameter $d_{\mathrm{sb}}$. In addition, geometrical properties are also integrated into $\chi_{\mathrm{b}}$ to form a dimensionless parameter reflecting the normalised contact area between the beam reinforcement and the surrounding concrete. As the concrete-reinforcement contact area has a direct influence on the magnitude of the bond forces transferring from the beam reinforcement into the concrete at the joint core, $\chi_{\mathrm{b}}$ is proposed as a parameter that can affect the joint shear strength. By considering its definition, it is easy to see that the joint aspect ratio $\left(\theta=h_{\mathrm{b}} / h_{\mathrm{c}}\right)$, which is considered as an influence factor of the joint shear strength, is integrated in $\chi_{b}$. Moreover, the conventional influence factor, the beam reinforcement ratio $\left(\rho_{\mathrm{sb}}=A_{\mathrm{sb}} / b_{\mathrm{b}} h_{\mathrm{b}}\right.$, where $A_{\mathrm{sb}}$ is the greater of area of the top or bottom beam bars) is also incorporated to a certain degree via the term $n_{\mathrm{b}} d_{\mathrm{sb}} / b_{\mathrm{b}} h_{\mathrm{b}}$ in this parameter. Details about the influence of both the beam bar index and other parameters on the joint shear strength are evaluated in the following sections.

\section{Parameters of influence in beam-column connections}

The 'key' influence parameters for the joint shear behaviour of beam-column connections were investigated by Kim and LaFave (2007). They found that concrete compressive strength $f_{\mathrm{c}}^{\prime}$, inplane geometry (interior, exterior or knee connections), dimensions of the beams and columns $\left(h_{\mathrm{b}}, b_{\mathrm{b}}, h_{\mathrm{c}}, b_{\mathrm{c}}\right)$, joint transverse reinforcement and beam reinforcement were among the influence parameters for the shear strength of beam-column connections. Besides the above parameters, bond condition - which is strongly influenced by the number and diameter of reinforcement bars and column axial stress are also known to affect joint shear strength.

Beam longitudinal bars passing or anchored in a joint core transfer a fraction of bond stress into the joint core and the remainder into the upper and lower columns. When the bond force of the beam bars is low, the fraction of bond force transferred into the columns is low, thus most of the shear force is claimed by the joint and slippage of the beam bars also occurs. The combination of these two unfavourable factors leads to a reduction in joint shear strength. Conversely, if the beam bars' bond force is high, the fraction of bond force transferred into the columns is significant; thus, a relatively small fraction of shear force is claimed by the joint and slippage of the beam bars would not occur, leading to an improvement in joint shear strength.

Among the parameters of influence, concrete compressive strength, in-plane geometry and the dimensions of the beam and column are the strongest, so their roles in joint shear strength have been mostly evaluated. The remaining parameters are still being debated. Some researchers (Bakir and Boduroğlu, 2002; Hegger et al., 2003; Kim and LaFave, 2008; Parker and Bullman, 1997; Paulay and Priestley, 1992; Sarsam and Phipps, 1985; Vollum and Newman, 1999) have proposed inconsistent contributions of joint shear reinforcement in the shear strength of beamcolumn joints while some (Marques and Jirsa, 1975, 1977; Pantazopoulou and Bonacci, 1992) have indicated that column axial load has no coherent effect on joint shear strength. Clyde et al. (2000) reported that column axial load helped to improve the joint shear strength while Park and Mosalam (2012) showed that joint shear strength is not clearly affected by column axial stress up to $0 \cdot 2 f_{\mathrm{c}}^{\prime}$.

\section{Models for shear strength of beam-column connections}

As mentioned earlier, strut and truss mechanisms are developed to resist joint shear forces, thus the joint shear strength $V_{\mathrm{jh}}$ is usually proposed to be composed of two components

1. $V_{\mathrm{jh}}=V_{\mathrm{ch}}+V_{\mathrm{sh}}$ 
$n_{\mathrm{b}}$ $d_{\mathrm{sb}}: m m$ $V_{\text {jh, test }}: \quad V_{\text {jh,model }}:$ $\mathrm{kN}$

Ehsani and Alameddine (1991)

$\begin{array}{llll}\text { HL8 } & 356 & 356 & 318\end{array}$

LL11

$356 \quad 356$

318

$$
508
$$

\section{HL11} $356 \quad 356 \quad 318$

Wong and Kuang (2008)

\begin{tabular}{|c|c|c|c|c|c|}
\hline Specimen & $b_{c}: m r$ & $h_{\mathrm{c}}: \mathrm{mm}$ & $b_{\mathrm{b}}: \mathrm{mm}$ & $h_{\mathrm{b}}: \mathrm{mm}$ & $f_{c}^{\prime}: M P a$ \\
\hline \multicolumn{6}{|c|}{ Ehsani and Alameddine (1991) } \\
\hline HL8 & 356 & 356 & 318 & 508 & 56 \\
\hline LL11 & 356 & 356 & 318 & 508 & 74 \\
\hline HL11 & 356 & 356 & 318 & 508 & 74 \\
\hline \multicolumn{6}{|c|}{ Wong and Kuang (2008) } \\
\hline BS-L-300 & 300 & 300 & 260 & 300 & 34 \\
\hline BS-L-450 & 300 & 300 & 260 & 450 & 31 \\
\hline BS-L-600 & 300 & 300 & 260 & 600 & 36 \\
\hline BS-L-V2 & 300 & 300 & 260 & 450 & 33 \\
\hline BS-L-V4 & 300 & 300 & 260 & 450 & 28 \\
\hline BS-L-H1 & 300 & 300 & 260 & 450 & 33 \\
\hline \multicolumn{6}{|c|}{ Tsonos (2007) } \\
\hline E1 & 200 & 200 & 200 & 300 & 22 \\
\hline G2 & 200 & 200 & 200 & 300 & 22 \\
\hline \multicolumn{6}{|c|}{ Clyde et al. (2000) } \\
\hline Test 2 & 305 & 457 & 305 & 406 & 46 \\
\hline Test 4 & 305 & 457 & 305 & 406 & 41 \\
\hline Test 5 & 305 & 457 & 305 & 406 & 37 \\
\hline Test 6 & 305 & 457 & 305 & 406 & 40 \\
\hline \multicolumn{6}{|c|}{ Kuang and Wong (2006) } \\
\hline BS-L & 300 & 300 & 260 & 450 & 31 \\
\hline$B S-U$ & 300 & 300 & 260 & 450 & 31 \\
\hline \multicolumn{6}{|c|}{ Tsonos et al. (1992) } \\
\hline$S 6^{\prime}$ & 200 & 200 & 200 & 300 & 29 \\
\hline \multicolumn{6}{|c|}{ Pantelides et al. (2002) } \\
\hline Test Unit 1 & 406 & 406 & 406 & 406 & 33 \\
\hline Test Unit 2 & 406 & 406 & 406 & 406 & 30 \\
\hline Test Unit 3 & 406 & 406 & 406 & 406 & 34 \\
\hline Test Unit 4 & 406 & 406 & 406 & 406 & 32 \\
\hline Test Unit 5 & 406 & 406 & 406 & 406 & 32 \\
\hline Test Unit 6 & 406 & 406 & 406 & 406 & 31 \\
\hline \multicolumn{6}{|c|}{ Chalioris et al. (2008) } \\
\hline$J A-0$ & 200 & 300 & 200 & 300 & 34 \\
\hline
\end{tabular}

\begin{tabular}{|c|c|c|c|c|c|}
\hline Specimen & $b_{c}: \mathrm{mm}$ & $h_{\mathrm{c}}: \mathrm{mm}$ & $b_{\mathrm{b}}: \mathrm{mm}$ & $h_{\mathrm{b}}: \mathrm{mm}$ & $f_{c}^{\prime}: M P a$ \\
\hline \multicolumn{6}{|c|}{ Ehsani and Alameddine (1991) } \\
\hline HL8 & 356 & 356 & 318 & 508 & 56 \\
\hline LL11 & 356 & 356 & 318 & 508 & 74 \\
\hline HL11 & 356 & 356 & 318 & 508 & 74 \\
\hline \multicolumn{6}{|c|}{ Wong and Kuang (2008) } \\
\hline BS-L-300 & 300 & 300 & 260 & 300 & 34 \\
\hline BS-L-450 & 300 & 300 & 260 & 450 & 31 \\
\hline BS-L-600 & 300 & 300 & 260 & 600 & 36 \\
\hline BS-L-V2 & 300 & 300 & 260 & 450 & 33 \\
\hline BS-L-V4 & 300 & 300 & 260 & 450 & 28 \\
\hline BS-L-H1 & 300 & 300 & 260 & 450 & 33 \\
\hline \multicolumn{6}{|c|}{ Tsonos (2007) } \\
\hline E1 & 200 & 200 & 200 & 300 & 22 \\
\hline G2 & 200 & 200 & 200 & 300 & 22 \\
\hline \multicolumn{6}{|c|}{ Clyde et al. (2000) } \\
\hline Test 2 & 305 & 457 & 305 & 406 & 46 \\
\hline Test 4 & 305 & 457 & 305 & 406 & 41 \\
\hline Test 5 & 305 & 457 & 305 & 406 & 37 \\
\hline Test 6 & 305 & 457 & 305 & 406 & 40 \\
\hline \multicolumn{6}{|c|}{ Kuang and Wong (2006) } \\
\hline$B S-L$ & 300 & 300 & 260 & 450 & 31 \\
\hline BS-U & 300 & 300 & 260 & 450 & 31 \\
\hline \multicolumn{6}{|c|}{ Tsonos et al. (1992) } \\
\hline S6' & 200 & 200 & 200 & 300 & 29 \\
\hline \multicolumn{6}{|c|}{ Pantelides et al. (2002) } \\
\hline Test Unit 1 & 406 & 406 & 406 & 406 & 33 \\
\hline Test Unit 2 & 406 & 406 & 406 & 406 & 30 \\
\hline Test Unit 3 & 406 & 406 & 406 & 406 & 34 \\
\hline Test Unit 4 & 406 & 406 & 406 & 406 & 32 \\
\hline Test Unit 5 & 406 & 406 & 406 & 406 & 32 \\
\hline Test Unit 6 & 406 & 406 & 406 & 406 & 31 \\
\hline \multicolumn{6}{|c|}{ Chalioris et al. (2008) } \\
\hline$J A-0$ & 200 & 300 & 200 & 300 & 34 \\
\hline
\end{tabular}

\begin{tabular}{|c|c|c|c|c|c|}
\hline Specimen & $b_{c}: \mathrm{mm}$ & $h_{\mathrm{c}}: \mathrm{mm}$ & $b_{\mathrm{b}}: \mathrm{mm}$ & $h_{\mathrm{b}}: \mathrm{mm}$ & $f_{c}^{\prime}: M P a$ \\
\hline \multicolumn{6}{|c|}{ Ehsani and Alameddine (1991) } \\
\hline HL8 & 356 & 356 & 318 & 508 & 56 \\
\hline LL11 & 356 & 356 & 318 & 508 & 74 \\
\hline HL11 & 356 & 356 & 318 & 508 & 74 \\
\hline \multicolumn{6}{|c|}{ Wong and Kuang (2008) } \\
\hline BS-L-300 & 300 & 300 & 260 & 300 & 34 \\
\hline BS-L-450 & 300 & 300 & 260 & 450 & 31 \\
\hline BS-L-600 & 300 & 300 & 260 & 600 & 36 \\
\hline BS-L-V2 & 300 & 300 & 260 & 450 & 33 \\
\hline BS-L-V4 & 300 & 300 & 260 & 450 & 28 \\
\hline BS-L-H1 & 300 & 300 & 260 & 450 & 33 \\
\hline \multicolumn{6}{|c|}{ Tsonos (2007) } \\
\hline E1 & 200 & 200 & 200 & 300 & 22 \\
\hline G2 & 200 & 200 & 200 & 300 & 22 \\
\hline \multicolumn{6}{|c|}{ Clyde et al. (2000) } \\
\hline Test 2 & 305 & 457 & 305 & 406 & 46 \\
\hline Test 4 & 305 & 457 & 305 & 406 & 41 \\
\hline Test 5 & 305 & 457 & 305 & 406 & 37 \\
\hline Test 6 & 305 & 457 & 305 & 406 & 40 \\
\hline \multicolumn{6}{|c|}{ Kuang and Wong (2006) } \\
\hline$B S-L$ & 300 & 300 & 260 & 450 & 31 \\
\hline BS-U & 300 & 300 & 260 & 450 & 31 \\
\hline \multicolumn{6}{|c|}{ Tsonos et al. (1992) } \\
\hline S6' & 200 & 200 & 200 & 300 & 29 \\
\hline \multicolumn{6}{|c|}{ Pantelides et al. (2002) } \\
\hline Test Unit 1 & 406 & 406 & 406 & 406 & 33 \\
\hline Test Unit 2 & 406 & 406 & 406 & 406 & 30 \\
\hline Test Unit 3 & 406 & 406 & 406 & 406 & 34 \\
\hline Test Unit 4 & 406 & 406 & 406 & 406 & 32 \\
\hline Test Unit 5 & 406 & 406 & 406 & 406 & 32 \\
\hline Test Unit 6 & 406 & 406 & 406 & 406 & 31 \\
\hline \multicolumn{6}{|c|}{ Chalioris et al. (2008) } \\
\hline$J A-0$ & 200 & 300 & 200 & 300 & 34 \\
\hline
\end{tabular}

BS-L-V2 $300 \quad 300 \quad 260$

BS-L-V4 $300 \quad 300 \quad 260$

BS-L-H1 $300 \quad 300 \quad 260$

Tsonos (2007)

\begin{tabular}{|c|c|c|c|c|c|}
\hline Specimen & $b_{c}: \mathrm{mm}$ & $h_{\mathrm{c}}: \mathrm{mm}$ & $b_{\mathrm{b}}: \mathrm{mm}$ & $h_{\mathrm{b}}: \mathrm{mm}$ & $f_{c}^{\prime}: M P a$ \\
\hline \multicolumn{6}{|c|}{ Ehsani and Alameddine (1991) } \\
\hline HL8 & 356 & 356 & 318 & 508 & 56 \\
\hline LL11 & 356 & 356 & 318 & 508 & 74 \\
\hline HL11 & 356 & 356 & 318 & 508 & 74 \\
\hline \multicolumn{6}{|c|}{ Wong and Kuang (2008) } \\
\hline BS-L-300 & 300 & 300 & 260 & 300 & 34 \\
\hline BS-L-450 & 300 & 300 & 260 & 450 & 31 \\
\hline BS-L-600 & 300 & 300 & 260 & 600 & 36 \\
\hline BS-L-V2 & 300 & 300 & 260 & 450 & 33 \\
\hline BS-L-V4 & 300 & 300 & 260 & 450 & 28 \\
\hline BS-L-H1 & 300 & 300 & 260 & 450 & 33 \\
\hline \multicolumn{6}{|c|}{ Tsonos (2007) } \\
\hline E1 & 200 & 200 & 200 & 300 & 22 \\
\hline G2 & 200 & 200 & 200 & 300 & 22 \\
\hline \multicolumn{6}{|c|}{ Clyde et al. (2000) } \\
\hline Test 2 & 305 & 457 & 305 & 406 & 46 \\
\hline Test 4 & 305 & 457 & 305 & 406 & 41 \\
\hline Test 5 & 305 & 457 & 305 & 406 & 37 \\
\hline Test 6 & 305 & 457 & 305 & 406 & 40 \\
\hline \multicolumn{6}{|c|}{ Kuang and Wong (2006) } \\
\hline$B S-L$ & 300 & 300 & 260 & 450 & 31 \\
\hline BS-U & 300 & 300 & 260 & 450 & 31 \\
\hline \multicolumn{6}{|c|}{ Tsonos et al. (1992) } \\
\hline S6' & 200 & 200 & 200 & 300 & 29 \\
\hline \multicolumn{6}{|c|}{ Pantelides et al. (2002) } \\
\hline Test Unit 1 & 406 & 406 & 406 & 406 & 33 \\
\hline Test Unit 2 & 406 & 406 & 406 & 406 & 30 \\
\hline Test Unit 3 & 406 & 406 & 406 & 406 & 34 \\
\hline Test Unit 4 & 406 & 406 & 406 & 406 & 32 \\
\hline Test Unit 5 & 406 & 406 & 406 & 406 & 32 \\
\hline Test Unit 6 & 406 & 406 & 406 & 406 & 31 \\
\hline \multicolumn{6}{|c|}{ Chalioris et al. (2008) } \\
\hline$J A-0$ & 200 & 300 & 200 & 300 & 34 \\
\hline
\end{tabular}

Clyde et al. (2000)

\begin{tabular}{|c|c|c|c|c|c|}
\hline Specimen & $b_{c}: \mathrm{mm}$ & $h_{\mathrm{c}}: \mathrm{mm}$ & $b_{\mathrm{b}}: \mathrm{mm}$ & $h_{\mathrm{b}}: \mathrm{mm}$ & $f_{c}^{\prime}: M P a$ \\
\hline \multicolumn{6}{|c|}{ Ehsani and Alameddine (1991) } \\
\hline HL8 & 356 & 356 & 318 & 508 & 56 \\
\hline LL11 & 356 & 356 & 318 & 508 & 74 \\
\hline HL11 & 356 & 356 & 318 & 508 & 74 \\
\hline \multicolumn{6}{|c|}{ Wong and Kuang (2008) } \\
\hline BS-L-300 & 300 & 300 & 260 & 300 & 34 \\
\hline BS-L-450 & 300 & 300 & 260 & 450 & 31 \\
\hline BS-L-600 & 300 & 300 & 260 & 600 & 36 \\
\hline BS-L-V2 & 300 & 300 & 260 & 450 & 33 \\
\hline BS-L-V4 & 300 & 300 & 260 & 450 & 28 \\
\hline BS-L-H1 & 300 & 300 & 260 & 450 & 33 \\
\hline \multicolumn{6}{|c|}{ Tsonos (2007) } \\
\hline E1 & 200 & 200 & 200 & 300 & 22 \\
\hline G2 & 200 & 200 & 200 & 300 & 22 \\
\hline \multicolumn{6}{|c|}{ Clyde et al. (2000) } \\
\hline Test 2 & 305 & 457 & 305 & 406 & 46 \\
\hline Test 4 & 305 & 457 & 305 & 406 & 41 \\
\hline Test 5 & 305 & 457 & 305 & 406 & 37 \\
\hline Test 6 & 305 & 457 & 305 & 406 & 40 \\
\hline \multicolumn{6}{|c|}{ Kuang and Wong (2006) } \\
\hline BS-L & 300 & 300 & 260 & 450 & 31 \\
\hline BS-U & 300 & 300 & 260 & 450 & 31 \\
\hline \multicolumn{6}{|c|}{ Tsonos et al. (1992) } \\
\hline$S 6^{\prime}$ & 200 & 200 & 200 & 300 & 29 \\
\hline \multicolumn{6}{|c|}{ Pantelides et al. (2002) } \\
\hline Test Unit 1 & 406 & 406 & 406 & 406 & 33 \\
\hline Test Unit 2 & 406 & 406 & 406 & 406 & 30 \\
\hline Test Unit 3 & 406 & 406 & 406 & 406 & 34 \\
\hline Test Unit 4 & 406 & 406 & 406 & 406 & 32 \\
\hline Test Unit 5 & 406 & 406 & 406 & 406 & 32 \\
\hline Test Unit 6 & 406 & 406 & 406 & 406 & 31 \\
\hline \multicolumn{6}{|c|}{ Chalioris et al. (2008) } \\
\hline$J A-0$ & 200 & 300 & 200 & 300 & 34 \\
\hline
\end{tabular}

Kuang and Wong (2006)

\begin{tabular}{|c|c|c|c|c|c|}
\hline Specimen & $b_{c}: m r$ & $h_{\mathrm{c}}: \mathrm{mm}$ & $b_{\mathrm{b}}: \mathrm{mm}$ & $h_{\mathrm{b}}: \mathrm{mm}$ & $f_{c}^{\prime}: M P a$ \\
\hline \multicolumn{6}{|c|}{ Ehsani and Alameddine (1991) } \\
\hline HL8 & 356 & 356 & 318 & 508 & 56 \\
\hline LL11 & 356 & 356 & 318 & 508 & 74 \\
\hline HL11 & 356 & 356 & 318 & 508 & 74 \\
\hline \multicolumn{6}{|c|}{ Wong and Kuang (2008) } \\
\hline BS-L-300 & 300 & 300 & 260 & 300 & 34 \\
\hline BS-L-450 & 300 & 300 & 260 & 450 & 31 \\
\hline BS-L-600 & 300 & 300 & 260 & 600 & 36 \\
\hline BS-L-V2 & 300 & 300 & 260 & 450 & 33 \\
\hline BS-L-V4 & 300 & 300 & 260 & 450 & 28 \\
\hline BS-L-H1 & 300 & 300 & 260 & 450 & 33 \\
\hline \multicolumn{6}{|c|}{ Tsonos (2007) } \\
\hline E1 & 200 & 200 & 200 & 300 & 22 \\
\hline G2 & 200 & 200 & 200 & 300 & 22 \\
\hline \multicolumn{6}{|c|}{ Clyde et al. (2000) } \\
\hline Test 2 & 305 & 457 & 305 & 406 & 46 \\
\hline Test 4 & 305 & 457 & 305 & 406 & 41 \\
\hline Test 5 & 305 & 457 & 305 & 406 & 37 \\
\hline Test 6 & 305 & 457 & 305 & 406 & 40 \\
\hline \multicolumn{6}{|c|}{ Kuang and Wong (2006) } \\
\hline BS-L & 300 & 300 & 260 & 450 & 31 \\
\hline$B S-U$ & 300 & 300 & 260 & 450 & 31 \\
\hline \multicolumn{6}{|c|}{ Tsonos et al. (1992) } \\
\hline$S 6^{\prime}$ & 200 & 200 & 200 & 300 & 29 \\
\hline \multicolumn{6}{|c|}{ Pantelides et al. (2002) } \\
\hline Test Unit 1 & 406 & 406 & 406 & 406 & 33 \\
\hline Test Unit 2 & 406 & 406 & 406 & 406 & 30 \\
\hline Test Unit 3 & 406 & 406 & 406 & 406 & 34 \\
\hline Test Unit 4 & 406 & 406 & 406 & 406 & 32 \\
\hline Test Unit 5 & 406 & 406 & 406 & 406 & 32 \\
\hline Test Unit 6 & 406 & 406 & 406 & 406 & 31 \\
\hline \multicolumn{6}{|c|}{ Chalioris et al. (2008) } \\
\hline$J A-0$ & 200 & 300 & 200 & 300 & 34 \\
\hline
\end{tabular}

$\begin{array}{llll}\text { S6' } & 200 & 200 & 200\end{array}$

Pantelides et al. (2002)

\begin{tabular}{|c|c|c|c|c|c|}
\hline Specimen & $b_{c}: \mathrm{mm}$ & $h_{\mathrm{c}}: \mathrm{mm}$ & $b_{\mathrm{b}}: \mathrm{mm}$ & $h_{\mathrm{b}}: \mathrm{mm}$ & $f_{c}^{\prime}: M P a$ \\
\hline \multicolumn{6}{|c|}{ Ehsani and Alameddine (1991) } \\
\hline HL8 & 356 & 356 & 318 & 508 & 56 \\
\hline LL11 & 356 & 356 & 318 & 508 & 74 \\
\hline HL11 & 356 & 356 & 318 & 508 & 74 \\
\hline \multicolumn{6}{|c|}{ Wong and Kuang (2008) } \\
\hline BS-L-300 & 300 & 300 & 260 & 300 & 34 \\
\hline BS-L-450 & 300 & 300 & 260 & 450 & 31 \\
\hline BS-L-600 & 300 & 300 & 260 & 600 & 36 \\
\hline BS-L-V2 & 300 & 300 & 260 & 450 & 33 \\
\hline BS-L-V4 & 300 & 300 & 260 & 450 & 28 \\
\hline BS-L-H1 & 300 & 300 & 260 & 450 & 33 \\
\hline \multicolumn{6}{|c|}{ Tsonos (2007) } \\
\hline E1 & 200 & 200 & 200 & 300 & 22 \\
\hline G2 & 200 & 200 & 200 & 300 & 22 \\
\hline \multicolumn{6}{|c|}{ Clyde et al. (2000) } \\
\hline Test 2 & 305 & 457 & 305 & 406 & 46 \\
\hline Test 4 & 305 & 457 & 305 & 406 & 41 \\
\hline Test 5 & 305 & 457 & 305 & 406 & 37 \\
\hline Test 6 & 305 & 457 & 305 & 406 & 40 \\
\hline \multicolumn{6}{|c|}{ Kuang and Wong (2006) } \\
\hline BS-L & 300 & 300 & 260 & 450 & 31 \\
\hline BS-U & 300 & 300 & 260 & 450 & 31 \\
\hline \multicolumn{6}{|c|}{ Tsonos et al. (1992) } \\
\hline$S 6^{\prime}$ & 200 & 200 & 200 & 300 & 29 \\
\hline \multicolumn{6}{|c|}{ Pantelides et al. (2002) } \\
\hline Test Unit 1 & 406 & 406 & 406 & 406 & 33 \\
\hline Test Unit 2 & 406 & 406 & 406 & 406 & 30 \\
\hline Test Unit 3 & 406 & 406 & 406 & 406 & 34 \\
\hline Test Unit 4 & 406 & 406 & 406 & 406 & 32 \\
\hline Test Unit 5 & 406 & 406 & 406 & 406 & 32 \\
\hline Test Unit 6 & 406 & 406 & 406 & 406 & 31 \\
\hline \multicolumn{6}{|c|}{ Chalioris et al. (2008) } \\
\hline$J A-0$ & 200 & 300 & 200 & 300 & 34 \\
\hline
\end{tabular}

\section{Chalioris et al. (2008)}

$\begin{array}{llll}\text { JA-0 } & 200 & 300 & 200 \\ \text { JB-0 } & 200 & 300 & 200\end{array}$

JB-s1 $200 \quad 300 \quad 200$

JCa-0 $100 \quad 200 \quad 100$

JCb-0 $100 \quad 200 \quad 100$

JCb-s1 $100 \quad 200 \quad 100$

JCb-s2 $100 \quad 200 \quad 100$

Karayannis and Sirkelis (2008)

$\begin{array}{lllllllllllll}\text { B0 } & 200 & 200 & 200 & 300 & 32 & 0.05 & 6 & 10 & 199 & 141 & 0.71 & \mathrm{~J} \\ \text { B1 } & 200 & 200 & 200 & 300 & 32 & 0.05 & 6 & 10 & 215 & 153 & 0.71 & \mathrm{~J} \\ \text { C0 } & 200 & 200 & 200 & 300 & 32 & 0.05 & 4 & 12 & 209 & 150 & 0.72 & \mathrm{~J} \\ \text { Alva et al. (2007) } & & & & & & & & & & & \\ \text { LVP4 } & 200 & 300 & 200 & 400 & 25 & 0.15 & 4 & 16 & 327 & 360 & 1.10 & \mathrm{~J}\end{array}$

Antonopoulos and Triantafillou (2003)

$\begin{array}{lrlllllllllll}\text { C1 } & 200 & 200 & 200 & 300 & 16 & 0 \cdot 12 & 3 & 14 & 116 & 98 & 0.85 & J \\ \text { C2 } & 200 & 200 & 200 & 300 & 19 & 0 \cdot 10 & 3 & 14 & 115 & 105 & 0.91 & J \\ \text { S-C } & 200 & 200 & 200 & 300 & 15 & 0 \cdot 12 & 3 & 14 & 123 & 103 & 0.84 & J\end{array}$

Table 1. Database of 98 exterior joints (continued on next page)

$\begin{array}{lllllll}0.07 & 4 & 29 & 986 & 869 & 0.88 & \mathrm{~J} \\ 0.03 & 4 & 25 & 768 & 865 & 1.13 & \mathrm{~J} \\ 0.06 & 4 & 29 & 967 & 954 & 0.99 & \mathrm{~J} \\ & & & & & & \\ 0.15 & 3 & 20 & 505 & 374 & 0.74 & \mathrm{~J} \\ 0.15 & 3 & 20 & 316 & 313 & 0.99 & \mathrm{~J} \\ 0.15 & 3 & 20 & 284 & 316 & 1.11 & \mathrm{~J} \\ 0.15 & 3 & 20 & 399 & 355 & 0.89 & \mathrm{~J} \\ 0.15 & 3 & 20 & 403 & 367 & 0.91 & \mathrm{~J} \\ 0.15 & 3 & 20 & 389 & 342 & 0.88 & \mathrm{~J} \\ & & & & & & \\ 0.18 & 3 & 14 & 232 & 195 & 0.84 & \mathrm{~J}\end{array}$

$\begin{array}{llllll}0.18 & 3 & 14 & 222 & 186 & 0.83\end{array}$

$\begin{array}{lllllll}0.10 & 4 & 29 & 947 & 946 & 1.00 \quad J\end{array}$

$\begin{array}{lllllll}0.25 & 4 & 29 & 982 & 1029 & 1.05 & \text { J }\end{array}$

$\begin{array}{lllllll}0.25 & 4 & 29 & 941 & 982 & 1.04 & \text { J }\end{array}$

$\begin{array}{lllllll}0.10 & 4 & 29 & 927 & 886 & 0.96 \quad J\end{array}$

$\begin{array}{lllllll}0.14 & 3 & 20 & 316 & 307 & 0.97 & J\end{array}$

$\begin{array}{lllllll}0.14 & 3 & 20 & 341 & 308 & 0.90 & J\end{array}$

$\begin{array}{lllllll}0.40 & 4 & 14 & 202 & 239 & 1.18\end{array}$

$\begin{array}{lllllll}0.10 & 4 & 29 & 872 & 733 & 0.84 & J\end{array}$

$\begin{array}{lllllll}0.25 & 4 & 29 & 833 & 836 & 1.00 & J\end{array}$

$\begin{array}{lllllll}0.10 & 4 & 29 & 826 & 743 & 0.90 & J\end{array}$

$\begin{array}{lllllll}0.25 & 4 & 29 & 927 & 855 & 0.92 & J\end{array}$

$\begin{array}{lllllll}0.10 & 4 & 29 & 770 & 717 & 0.93 & J\end{array}$

$\begin{array}{lllllll}0.25 & 4 & 29 & 851 & 847 & 1.00 & \text { J }\end{array}$

$\begin{array}{lllllll}0.05 & 4 & 12 & 218 & 255 & 1.17 & \text { J }\end{array}$

$\begin{array}{lllllll}0.05 & 6 & 10 & 201 & 251 & 1.25 & \text { J }\end{array}$

$\begin{array}{lllllll}0.05 & 6 & 10 & 219 & 258 & 1.18 & J\end{array}$

$\begin{array}{lllllll}0.10 & 3 & 8 & 66 & 66 & 1.01 & J\end{array}$

$\begin{array}{lllllll}0.10 & 3 & 10 & 84 & 76 & 0.90 & \mathrm{~J}\end{array}$

$\begin{array}{llllll}0.10 & 3 & 10 & 97 & 83 & 0.86\end{array}$

$\begin{array}{lllllll}0.10 & 3 & 10 & 88 & 91 & 1.03 & \text { J }\end{array}$


Specimen $\quad b_{\mathrm{c}}: \mathrm{mm} \quad h_{\mathrm{c}}: \mathrm{mm} \quad b_{\mathrm{b}}: \mathrm{mm} \quad h_{\mathrm{b}}: \mathrm{mm} \quad f_{\mathrm{c}}^{\prime}: \mathrm{MPa}$ $\frac{N}{b_{c} h_{c} f_{c}^{\prime}}$

El-Amoury and Ghobarah (2002)

Ghobarah and Said (2001)

T2 $250 \quad 400$

T-BS3

$250 \quad 400 \quad 250$

Shrestha et al. (2009)

$300 \quad 300$

Fisher and Sezen (2011)

B-1-RC

R1

S1

$1 \mathrm{~B}$

2B

$5 B$

4

LL8

LH8

$\mathrm{HH} 8$

$\mathrm{HH} 11$

LL14

LH14

$\mathrm{HH} 14$

OTO

3T3

2T4

152

152

$$
\text { 200 }
$$

250

$200 \quad 250$

$200 \quad 250 \quad 200$

$300 \quad 300 \quad 259$

$300 \quad 300 \quad 259$

$340 \quad 340 \quad 300$

$\begin{array}{lll}300 & 300 \quad 259\end{array}$

$355 \cdot 6$

355.6

$355 \cdot 6$

355.6

$355 \cdot 6$

355.6

$355 \cdot 6$

317.5

$420 \quad 420 \quad 320$

$420 \quad 420 \quad 320$

$1 \mathrm{~T} 44$

$420 \quad 420 \quad 320$

$420 \quad 420 \quad 320$

Wong and Kuang (2008)

\begin{tabular}{|c|c|c|c|c|}
\hline BS-L-H2 & 300 & 300 & 260 & 450 \\
\hline JCa-s1 & 100 & 200 & 100 & 200 \\
\hline JCa-s2 & 100 & 200 & 100 & 200 \\
\hline$J C-2$ & 500 & 500 & 350 & 500 \\
\hline JC-No.11 & 650 & 520 & 450 & 505 \\
\hline \multicolumn{5}{|c|}{ Karayannis and Sirkelis (2008) } \\
\hline A1 & 200 & 200 & 200 & 300 \\
\hline A2 & 200 & 200 & 200 & 300 \\
\hline \multicolumn{5}{|c|}{ Karayannis et al. (2008) } \\
\hline A0 & 200 & 200 & 200 & 300 \\
\hline$C 2$ & 200 & 200 & 200 & 300 \\
\hline \multicolumn{5}{|c|}{ Hwang et al. (2004) } \\
\hline 28-0Т0 & 550 & 550 & 380 & 500 \\
\hline LVP2 & 200 & 300 & 200 & 400 \\
\hline LVP3 & 200 & 300 & 200 & 400 \\
\hline LVP5 & 200 & 300 & 200 & 400 \\
\hline Unit RC-1 & 230 & 230 & 200 & 330 \\
\hline AJ1s & 200 & 200 & 200 & 300 \\
\hline
\end{tabular}

$400 \quad 31$

400

$400 \quad 30$

$450 \quad 26$

203

30

400

400

400

480

439

480

439

508

508

508

508

508

508

508

450

450

450

450

26

$$
30
$$

28

34

35

24

67

56

56

56

74

94

94

94

67

69

71

73

\section{2}

21

21

60

31

36

36

0.0

0.05

$0 \cdot 20 \quad 4 \quad 20$

$0 \cdot 10 \quad 4 \quad 20$

420

536

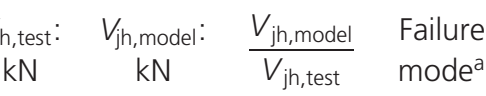

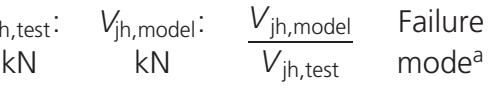

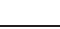




\begin{tabular}{|c|c|c|c|c|c|c|c|c|c|c|c|c|}
\hline Specimen & $b_{c}: m m$ & $h_{c}: \mathrm{mm}$ & $b_{\mathrm{b}}: \mathrm{mm}$ & $h_{\mathrm{b}}: \mathrm{mm}$ & $f_{c}^{\prime}: M P a$ & $\frac{N}{b_{c} h_{c} f_{c}^{\prime}}$ & $n_{\mathrm{b}}$ & $d_{\mathrm{sb}}: \mathrm{mm}$ & $\begin{array}{c}V_{\text {jh, test }}: \\
\text { kN }\end{array}$ & $\begin{array}{c}V_{\text {jh, model }}: \\
\text { kN }\end{array}$ & $\frac{V_{\text {jh,model }}}{V_{\text {jh,test }}}$ & $\begin{array}{l}\text { Failure } \\
\text { mode }^{a}\end{array}$ \\
\hline NS & 167 & 167 & 134 & 200 & 37 & 0.00 & 4 & 10 & 120 & 96 & $0 \cdot 80$ & BJ \\
\hline SD & 167 & 167 & 134 & 200 & 37 & 0.00 & 4 & 10 & 114 & 101 & $0 \cdot 88$ & BJ \\
\hline $\mathrm{T} 1$ & 250 & 400 & 250 & 400 & 31 & $0 \cdot 19$ & 4 & 20 & 527 & 537 & 1.02 & BJ \\
\hline $1 s t$ & 350 & 350 & 350 & 400 & 24 & 0.00 & 4 & 18 & 400 & 463 & $1 \cdot 16$ & BJ \\
\hline $2 n d$ & 350 & 350 & 350 & 400 & 20 & 0.00 & 4 & 18 & 425 & 429 & 1.01 & BJ \\
\hline \multicolumn{13}{|c|}{ Fisher and Sezen (2011) } \\
\hline$C-2-R C$ & 152 & 152 & 152 & 203 & 30 & 0.00 & 3 & 10 & 102 & 102 & 1.00 & BJ \\
\hline $\mathrm{E}-1-\mathrm{RC}$ & 152 & 152 & 152 & 203 & 30 & 0.00 & 3 & 12 & 113 & 107 & 0.95 & BJ \\
\hline UNIT A & 330 & 380 & 255 & 460 & 22 & 0.07 & 3 & 28 & 547 & 548 & 1.00 & BJ \\
\hline Q3 & 200 & 250 & 200 & 400 & 27 & 0.00 & 2 & 20 & 211 & 222 & 1.05 & BJ \\
\hline S3 & 200 & 250 & 200 & 400 & 30 & 0.00 & 2 & 20 & 198 & 229 & $1 \cdot 16$ & BJ \\
\hline Specimen I & 406 & 406 & 356 & 457 & 28 & 0.00 & 4 & 25 & 1040 & 846 & 0.81 & BJ \\
\hline \multicolumn{13}{|c|}{ Kaku and Asakusa (1991) } \\
\hline NO 3 & 220 & 220 & 160 & 220 & 42 & 0.00 & 4 & 13 & 217 & 208 & 0.96 & BJ \\
\hline NO 4 & 220 & 220 & 160 & 220 & 45 & 0.17 & 4 & 13 & 239 & 254 & 1.06 & BJ \\
\hline NO 5 & 220 & 220 & 160 & 220 & 37 & 0.09 & 4 & 13 & 221 & 210 & 0.95 & BJ \\
\hline NO 6 & 220 & 220 & 160 & 220 & 40 & 0.00 & 4 & 13 & 209 & 196 & 0.94 & BJ \\
\hline NO 9 & 220 & 220 & 160 & 220 & 41 & 0.00 & 4 & 13 & 236 & 232 & 0.98 & BJ \\
\hline NO 11 & 220 & 220 & 160 & 220 & 42 & 0.08 & 4 & 13 & 231 & 248 & 1.08 & BJ \\
\hline NO 12 & 220 & 220 & 160 & 220 & 35 & 0.00 & 4 & 13 & 207 & 210 & 1.01 & BJ \\
\hline NO 13 & 220 & 220 & 160 & 220 & 46 & -0.04 & 4 & 13 & 209 & 236 & $1 \cdot 13$ & BJ \\
\hline NO 14 & 220 & 220 & 160 & 220 & 41 & 0.08 & 4 & 13 & 226 & 226 & 1.00 & BJ \\
\hline NO 15 & 220 & 220 & 160 & 220 & 40 & 0.08 & 4 & 13 & 230 & 229 & 0.99 & BJ \\
\hline \multicolumn{13}{|c|}{ Park and Paulay (1974) } \\
\hline S4 & 330 & 381 & 254 & 457 & 21 & 0.00 & 2 & 29 & 317 & 369 & $1 \cdot 17$ & BJ \\
\hline Average & & & & & & & & & & & 1.00 & \\
\hline $\mathrm{CoV}$ & & & & & & & & & & & $0 \cdot 147$ & \\
\hline
\end{tabular}

a J, joint shear failure; BJ, joint shear failure with yielding of beam reinforcement

Table 1. (continued)

where $V_{\text {ch }}$ and $V_{\text {sh }}$ are the contributions of the concrete strut and truss mechanisms respectively. The first component is related to the concrete strength $f_{\mathrm{c}}^{\prime}$ and the second term relates to the horizontal and vertical joint reinforcement.

ACI 352R-02 (ACI, 2002) and Architectural Institute of Japan design guidelines AIJ (1999) ignore the contribution of $V_{\mathrm{sh}}$, thus the joint shear strength is expressed as a function of concrete compressive strength and the joint geometry. The form of the expression is

\section{2. $\quad V_{\mathrm{jh}}=k_{1} k_{2}\left(f_{\mathrm{c}}^{\prime}\right)^{m} A_{\mathrm{jh}}$}

where $k_{1}$ and $k_{2}$ are joint shear factors that depend on the joint geometry (in ACI 352R-02, $k_{1} \times k_{2}$ is equal to the joint shear strength factor, $\left.\gamma_{\mathrm{ACI}}\right), A_{\mathrm{jh}}$ is the effective joint area and $m=0.5$ in the ACI model and $m=0.7$ in the AJI model.
In NZS-3101 (SANZ, 1995), the contribution of the truss mechanism is somehow considered but a clear contribution of $V_{\text {sh }}$ on joint shear strength is not available. The formulation of joint shear strength in NZS-3101 is

3. $\quad V_{\mathrm{jh}}=\frac{f_{\mathrm{jhy}} A_{\mathrm{sjh}}}{6 \alpha_{\mathrm{NZS}} f_{\mathrm{by}} A_{\mathrm{sb}}}\left(f_{\mathrm{c}}^{\prime}\right)^{m} A_{\mathrm{jh}} \leqslant 0 \cdot 2\left(f_{\mathrm{c}}^{\prime}\right) A_{\mathrm{jh}}$

in which $m=1, f_{\text {jhy }}$ and $A_{\text {sjh }}$ are the yield strength and the total cross-sectional area of the horizontal joint transverse reinforcement respectively, $f_{\text {by }}$ and $A_{\mathrm{sb}}$ are the yield strength and the greater of the area of top or bottom beam bars respectively and $\alpha_{\mathrm{NZS}}$ is a factor that reflects the influence of both joint geometry and column axial load.

Paulay and Priestley (1992) proposed a theoretical joint shear strength model in which the contribution of the concrete strut $V_{\text {ch }}$ depends on the area and yielding strength of the beam's long- 
Specimen $\quad b_{c}: m m \quad h_{c}: m m \quad b_{b}: m m \quad h_{b}: m m \quad f_{c}^{\prime}: M P a$ $\frac{N}{b_{c} h_{c} f_{c}^{\prime}}$ $n_{\mathrm{b}} \quad d_{\mathrm{sb}}: \mathrm{m}$

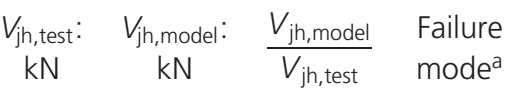
Meinheit and Jirsa (1977)

I $330 \quad 457$

IV

$\checkmark$

VII

XIII

XIV

457

J-10 $300 \quad 300$

J-11

300300

$$
\begin{aligned}
& 279 \\
& 279 \\
& 279 \\
& 406 \\
& 279 \\
& 406 \\
& 279 \\
& 406
\end{aligned}
$$

$330 \quad 457$

$330 \quad 457$

$\mathrm{SHC} 1$

$\mathrm{SHC2}$

127

SOC3

240

240

240

127

$127 \quad 203$

$127 \quad 203$

Kitayama et al. (1991)

A1

Au et al. (2005)

E-0.0

$\mathrm{H}-\mathrm{O} \cdot \mathrm{O}$

$\mathrm{E}-0 \cdot 3$

Lee et al. (2010)

J10 $400 \quad 400$

OKJ3

OKJ5

$$
300
$$

300

300

200

300

300

Dhakal et al. (2005)

C1PD

$$
350
$$

400

Morita et al. (1999)

No 2

$$
350
$$

$500 \quad 300$

$400 \quad 300$

350

250

457

\section{7}

457

457

457

457

457

457

26
42
27
36
36
37
41
33

300

300

300

81

39

39

57

60

47

203

\section{6}

No 4

350

350

350

250

250

350

350

250

No 6

$350 \quad 350$

250

Ishibashi (1993)

$\begin{array}{llllll}\text { D19-S1 } & 400 & 400 & 260 & 400 & 44 \\ \text { D19-S2 } & 400 & 400 & 260 & 400 & 43 \\ \text { D25-S3 } & 400 & 400 & 260 & 400 & 48 \\ \text { D25-S4 } & 400 & 400 & 260 & 400 & 48 \\ \text { D29-S5 } & 400 & 400 & 260 & 400 & 48\end{array}$

Wang and Hsu (2009)

Ko-J1 $300 \quad 300$

Ho-J11 $400 \quad 400$

Au et al. (2005)

$\mathrm{E}-0 \cdot 0$ 300
$300 \quad 500 \quad 32$

400
$0.40 \quad 3 \quad 25$

$0.25 \quad 3 \quad 25$

$0.39 \quad 3 \quad 25$

$0.30 \quad 3 \quad 25$

$0.04 \quad 3 \quad 25$

$0.47 \quad 3 \quad 25$

$0.25 \quad 3 \quad 25$

$0.32 \quad 3 \quad 25$

$0 \cdot 11 \quad 8 \quad 13$

$0.12 \quad 9 \quad 13$

$0 \cdot 12 \quad 9 \quad 19$

$\begin{array}{lll}0.05 & 2 & 12\end{array}$

$0.04 \quad 2 \quad 12$

$\begin{array}{lll}0.05 & 2 & 12\end{array}$

$\begin{array}{lll}0.08 & 8 & 13\end{array}$

$0.06 \quad 8 \quad 13$

$0.00 \quad 4 \quad 16$

$0.00 \quad 4 \quad 16$

$0.35 \quad 4 \quad 16$

$0.19 \quad 3 \quad 25$

$\begin{array}{lll}0.12 & 10 \quad 13\end{array}$

$\begin{array}{lll}0.12 & 10 & 13\end{array}$

$0.12 \quad 8 \quad 13$

$\begin{array}{lll}0.12 & 5 & 32\end{array}$

$0.12 \quad 6 \quad 32$

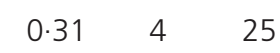

$\begin{array}{lll}-0.31 \quad 4 & 25\end{array}$

$0.30 \quad 4 \quad 25$

$\begin{array}{lll}0.31 \quad 7 & 16\end{array}$

$\begin{array}{lll}-0.31 \quad 7 & 16\end{array}$

$\begin{array}{lll}0.00 & 5 & 19\end{array}$

$0.00 \quad 3 \quad 19$

$0.00 \quad 3 \quad 25$

$0.00 \quad 3 \quad 25$

$\begin{array}{lll}0.00 & 3 & 29\end{array}$

$0.14 \quad 4 \quad 25$

$\begin{array}{lll}0.00 \quad 4 & 19\end{array}$

$0.00 \quad 4 \quad 16$

1090
1597
1228
1455
1530
1646
1468
1948

$1557 \quad 1528$

$1539 \quad 1199$

516506

536

576

503

491

840

853

629

1042

1119

$1075 \quad 1166$

$1204 \quad 1178$

$1111 \quad 1137$

888

1139

1180

1.28

0.94

795

941

156

164

163

873

857

186

193

174

$1 \cdot 10$

0.91

$1 \cdot 19$

$1 \cdot 18$

493

429

628

545

826

573

573

575

568

662

750

679

763

0.91

0.88

814

855

1.05

Table 2. Database of 73 interior joints (continued on next page) 


\begin{tabular}{|c|c|c|c|c|c|c|c|c|c|c|c|c|}
\hline Specimen & $b_{c}: m m$ & $h_{\mathrm{c}}: \mathrm{mm}$ & $b_{\mathrm{b}}: \mathrm{mm}$ & $h_{\mathrm{b}}: \mathrm{mm}$ & $f_{c}^{\prime}: M P a$ & $\frac{N}{b_{c} h_{c} f_{c}^{\prime}}$ & $n_{\mathrm{b}}$ & $d_{\mathrm{sb}}: \mathrm{mm}$ & $\begin{array}{l}V_{\text {jh,test }}: \\
\text { kN }\end{array}$ & $\begin{array}{c}V_{\text {jh, model }}: \\
\text { kN }\end{array}$ & $\frac{V_{\text {jh, model }}}{V_{\text {jh,test }}}$ & $\begin{array}{l}\text { Failure } \\
\text { mode }^{a}\end{array}$ \\
\hline$E-0 \cdot 3$ & 300 & 300 & 250 & 300 & 39 & 0.26 & 4 & 16 & 1038 & 991 & 0.96 & J \\
\hline $\mathrm{H}-0 \cdot 0$ & 300 & 300 & 250 & 300 & 43 & 0.00 & 4 & 16 & 1081 & 974 & 0.90 & $J$ \\
\hline $\mathrm{H}-0 \cdot 3$ & 300 & 300 & 250 & 300 & 38 & 0.26 & 4 & 16 & 378 & 384 & $1 \cdot 02$ & $J$ \\
\hline \multicolumn{13}{|c|}{ Meinheit and Jirsa (1977) } \\
\hline $\mathrm{VI}$ & 330 & 457 & 279 & 457 & 37 & 0.48 & 3 & 25 & 414 & 451 & 1.09 & BJ \\
\hline XII & 330 & 457 & 279 & 457 & 35 & $0 \cdot 30$ & 3 & 25 & 876 & 940 & $1 \cdot 07$ & BJ \\
\hline \multicolumn{13}{|c|}{ Otani et al. (1984) } \\
\hline J1 & 300 & 300 & 200 & 300 & 26 & 0.08 & 3 & 19 & 1180 & 1086 & 0.92 & BJ \\
\hline 12 & 300 & 300 & 200 & 300 & 24 & 0.08 & 3 & 19 & 1458 & 1328 & 0.91 & BJ \\
\hline 13 & 300 & 300 & 200 & 300 & 24 & 0.08 & 3 & 19 & 1180 & 1086 & 0.92 & BJ \\
\hline J4 & 300 & 300 & 200 & 300 & 26 & 0.23 & 3 & 19 & 1180 & 1113 & 0.94 & BJ \\
\hline J5 & 300 & 300 & 200 & 300 & 29 & 0.07 & 3 & 19 & 1031 & 964 & 0.94 & BJ \\
\hline \multicolumn{13}{|c|}{ Durrani and Wight (1985) } \\
\hline $\mathrm{X} 1$ & 362 & 362 & 279 & 419 & 34 & 0.05 & 4 & 22 & 798 & 635 & 0.80 & BJ \\
\hline$x 2$ & 362 & 362 & 279 & 419 & 34 & 0.06 & 4 & 22 & 660 & 635 & 0.96 & BJ \\
\hline X3 & 362 & 362 & 279 & 419 & 31 & 0.05 & 3 & 22 & 844 & 630 & 0.75 & BJ \\
\hline \multicolumn{13}{|c|}{ Oka and Shiohara (1992) } \\
\hline$J-1$ & 300 & 300 & 240 & 300 & 81 & 0.11 & 9 & 13 & 737 & 596 & $0 \cdot 81$ & BJ \\
\hline $\mathrm{J}-4$ & 300 & 300 & 240 & 300 & 73 & $0 \cdot 13$ & 10 & 13 & 1273 & 1436 & $1 \cdot 13$ & BJ \\
\hline$J-5$ & 300 & 300 & 240 & 300 & 73 & 0.13 & 9 & 13 & 1045 & 1203 & $1 \cdot 15$ & BJ \\
\hline J-6 & 300 & 300 & 240 & 300 & 79 & $0 \cdot 12$ & 9 & 13 & 871 & 988 & $1 \cdot 14$ & BJ \\
\hline J-7 & 300 & 300 & 240 & 300 & 79 & 0.12 & 7 & 13 & 822 & 683 & 0.83 & BJ \\
\hline $\mathrm{J}-8$ & 300 & 300 & 240 & 300 & 79 & $0 \cdot 12$ & 9 & 19 & 920 & 993 & 1.08 & BJ \\
\hline \multicolumn{13}{|c|}{ Kitayama et al. (1991) } \\
\hline C1 & 300 & 300 & 200 & 300 & 26 & 0.08 & 12 & 10 & 865 & 991 & $1 \cdot 15$ & BJ \\
\hline B1 & 300 & 300 & 200 & 300 & 25 & 0.08 & 8 & 13 & 810 & 686 & 0.85 & BJ \\
\hline B3 & 300 & 300 & 200 & 300 & 25 & 0.08 & 8 & 13 & 1509 & 1318 & $0 \cdot 87$ & BJ \\
\hline \multicolumn{13}{|c|}{ Lu et al. (2012) } \\
\hline$J 1-1$ & 400 & 400 & 250 & 400 & 30 & 0.04 & 4 & 20 & 973 & 1151 & $1 \cdot 18$ & BJ \\
\hline $\mathrm{J} 1-4$ & 400 & 400 & 250 & 400 & 30 & 0.04 & 3 & 25 & 1542 & 1272 & $0 \cdot 82$ & BJ \\
\hline \multicolumn{13}{|c|}{ Leon (1990) } \\
\hline $\mathrm{BCJ} 2$ & 254 & 254 & 203 & 305 & 30 & 0.00 & 4 & 13 & 1053 & 1272 & $1 \cdot 21$ & BJ \\
\hline $\mathrm{BCJ} 3$ & 254 & 305 & 203 & 305 & 27 & 0.00 & 4 & 13 & 1309 & 1322 & $1 \cdot 01$ & BJ \\
\hline \multicolumn{13}{|c|}{ Noguchi and Kashiwazaki (1992) } \\
\hline OKJ1 & 300 & 300 & 200 & 300 & 78 & $0 \cdot 12$ & 9 & 13 & 411 & 501 & $1 \cdot 22$ & BJ \\
\hline \multicolumn{13}{|c|}{ Noguchi and Kurusu (1988) } \\
\hline OKJ4 & 300 & 300 & 200 & 300 & 78 & 0.12 & 9 & 13 & 467 & 537 & $1 \cdot 15$ & BJ \\
\hline NO.1 & 300 & 300 & 200 & 300 & 34 & 0.06 & 12 & 10 & 484 & 560 & $1 \cdot 16$ & BJ \\
\hline NO.2 & 300 & 300 & 200 & 300 & 34 & 0.06 & 10 & 10 & 759 & 606 & 0.80 & BJ \\
\hline NO.3 & 300 & 300 & 200 & 300 & 34 & 0.06 & 6 & 13 & 893 & 868 & 0.97 & BJ \\
\hline NO.4 & 300 & 300 & 200 & 300 & 34 & 0.06 & 5 & 13 & 750 & 644 & 0.86 & BJ \\
\hline \multicolumn{13}{|c|}{ Supaviriyakit and Pimanmas (2008) } \\
\hline J1 & 200 & 350 & 175 & 300 & 26 & 0.11 & 6 & 12 & 814 & 803 & 0.99 & BJ \\
\hline$J 2 A$ & 200 & 350 & 175 & 300 & 29 & $0 \cdot 10$ & 6 & 12 & 869 & 770 & $0 \cdot 89$ & BJ \\
\hline J3B & 200 & 350 & 175 & 300 & 24 & $0 \cdot 12$ & 6 & 12 & 744 & 854 & $1 \cdot 15$ & BJ \\
\hline Average & & & & & & & & & & & $1 \cdot 00$ & \\
\hline $\mathrm{CoV}$ & & & & & & & & & & & $0 \cdot 140$ & \\
\hline
\end{tabular}

a J, joint shear failure; BJ, joint shear failure with yielding of beam reinforcement 


\begin{tabular}{|c|c|c|c|c|c|}
\hline & \multirow[t]{2}{*}{ Definition } & \multicolumn{2}{|c|}{ Exterior joints } & \multicolumn{2}{|c|}{ Interior joints } \\
\hline & & Minimum & Maximum & Minimum & Maximum \\
\hline Concrete compressive strength $f_{\mathrm{c}}^{\prime}: \mathrm{MPa}$ & & 15 & 94 & 22 & 118 \\
\hline Normalised column axial stress & $\frac{N}{b_{c} h_{c} f_{c}^{\prime}}$ & -0.04 & 0.40 & -0.31 & $0 \cdot 48$ \\
\hline Normalised horizontal joint shear reinforcement & $\chi_{\mathrm{jh}}=\frac{A_{\mathrm{sh} h} f_{\mathrm{jhh}}}{b_{\mathrm{j}} h_{\mathrm{c}}\left(f_{\mathrm{c}}^{\prime}\right)^{0.5}}$ & 0.00 & $1 \cdot 11$ & 0.00 & $1 \cdot 23$ \\
\hline Normalised vertical joint shear reinforcement & $\chi_{\mathrm{jv}}=\frac{A_{\mathrm{sj}} f_{\mathrm{jyy}}}{b_{\mathrm{j}} h_{\mathrm{c}}\left(f_{\mathrm{c}}^{\prime}\right)^{0.5}}$ & 0.00 & 1.63 & 0.00 & $3 \cdot 20$ \\
\hline Normalised joint shear reinforcement & $\chi_{\mathrm{j}}=\chi_{\mathrm{jh}}+\chi_{\mathrm{jv}}$ & 0.00 & 2.45 & 0.00 & 3.50 \\
\hline Column depth to beam height ratio & $h_{\mathrm{c}} / h_{\mathrm{b}}$ & 0.50 & $1 \cdot 13$ & 0.67 & 1.00 \\
\hline Beam bar diameter to column depth ratio & $d_{b} / h_{c}$ & 0.03 & 0.09 & 0.03 & 0.08 \\
\hline Beam bar index & $\chi_{\mathrm{b}}=\frac{n_{\mathrm{b}} d_{\mathrm{sb}}}{b_{\mathrm{b}}} \frac{h_{\mathrm{c}}}{h_{\mathrm{b}}}$ & 0.07 & 0.51 & 0.13 & 0.71 \\
\hline
\end{tabular}

Table 3. Definitions and ranges of parameters in the collected database

itudinal reinforcement, the concrete compressive strength, column axial load and the joint geometry. The contribution of the truss mechanism is simply determined as $V_{\mathrm{sh}}=A_{\text {sih }} f_{\text {jhy }}$. A similar contribution of the truss mechanism on joint shear strength was proposed by Vollum and Newman (1999), but their model additionally accounted for the influence of $h_{\mathrm{b}} / h_{\mathrm{c}}$.

Bakir and Boduroğlu (2002) proposed an empirical model in which $V_{\text {ch }}$ depends on concrete strength, details of the beam reinforcement and joint aspect ratio $h_{\mathrm{b}} / h_{\mathrm{c}}$. In their model, the contribution of the truss mechanism is determined as $V_{\mathrm{sh}}=\alpha_{\mathrm{j}} A_{\mathrm{sjh}}$ $f_{\text {jhy }}$, where $\alpha_{\mathrm{j}}$ depends on the joint shear reinforcement ratio. A similar form was proposed by Hegger et al. (2003) for determining $V_{\text {sh }}$, but the parameter $\alpha_{\mathrm{j}}$ was supposed to be influenced by the pattern of the beam bars being anchored and details of the joint shear reinforcement. Different from other models, this model accounted for the influence of the column reinforcement ratio.

Using the Bayesian parameter estimation method, Kim et al. (2009) developed an empirical joint shear strength model. The distinctive feature of this model is that the joint shear strength is given in terms of multiplication of the influence parameters

$$
\text { 4. } \quad V_{\mathrm{jh}}=1 \cdot 31 \alpha_{\mathrm{t}} \beta_{\mathrm{t}} \eta_{\mathrm{t}}(\mathrm{JI})^{0 \cdot 15}(\mathrm{BI})^{0 \cdot 3}\left(f_{\mathrm{c}}^{\prime}\right)^{0 \cdot 75} A_{\mathrm{jh}}
$$

in which $\alpha_{\mathrm{t}}$ and $\beta_{\mathrm{t}}$ are parameters for describing in-plane and out-of-plane geometry respectively, $\eta_{\mathrm{t}}$ is a parameter to account for the influence of beam eccentricity, JI is the joint transverse reinforcement index (depending mostly on the volumetric joint shear reinforcement ratio) and $\mathrm{BI}$ is the beam reinforcement index (depending mostly on the beam reinforcement ratio $\rho_{\mathrm{sb}}$ ). A limitation of this model is that column axial load was not taken into account as a possible influence parameter for joint shear strength during development of the model. This is because, in the Bayesian parameter estimation model, all parameters should be non-zero (Kim and LaFave, 2008) and some experimental tests had zero column axial load.

Based on the strut-and-tie approach, Hwang and Lee (2002) developed a theoretical joint shear strength model in which the joint shear strength was determined as

5. $V_{\mathrm{jh}}=K \xi f_{\mathrm{c}}^{\prime} A_{\mathrm{str}} \cos \theta$

where $K$ is a factor accounting for the contribution of horizontal and vertical joint shear reinforcement, $\zeta\left(=3 \cdot 35 /\left(f_{\mathrm{c}}^{\prime}\right)^{0 \cdot 5} \leqslant 0 \cdot 52\right)$ is a softening coefficient, $A_{\text {str }}$ is the effective area of the diagonal strut depending on column axial load and dimensions of the joint and $\theta$ is the angle of inclination of the diagonal compression strut with respect to the longitudinal direction of the beam. The model of Hwang and Lee (2002) can clearly illustrate the strutand-tie mechanism in the joint core but the joint shear prediction is known to be very sensitive to $A_{\text {str }}$, which is difficult to determine accurately.

Based on the average stress approach, Tsonos (2007) developed a theoretical model for the shear strength of beam-column joints. In the model, a biaxial compression-tension failure envelope was adopted to predict the joint shear strength. However, this model exaggerated the role of joint shear reinforcement in confining the concrete at the strut, while its influence on the truss mechanism 
was ignored. Using a similar method, Wang et al. (2012) developed a theoretical model for the shear strength of beamcolumn joints. However, the limitation of their model is that the role of joint shear reinforcement was exaggerated by assuming that the tensile strength of the concrete and the joint shear reinforcement is reached simultaneously. The model of Wang et al. (2012) is

6.

$$
V_{\mathrm{jh}}=\beta \frac{1-\left(\sin ^{2} \alpha / f_{\mathrm{t}, \mathrm{n}}-0 \cdot 8 \cos ^{2} \alpha / f_{\mathrm{c}}^{\prime}\right) \sigma_{\mathrm{y}}}{\left(1 / f_{\mathrm{t}, \mathrm{n}}+0 \cdot 8 / f_{\mathrm{c}}^{\prime}\right) \sin 2 \alpha} A_{\mathrm{jh}}
$$

where $\beta=1 \cdot 0$ and 0.8 for interior and exterior joints respectively, $\alpha$ is the inclination of the diagonal strut, $f_{\mathrm{t}, \mathrm{n}}$ is the nominal tensile strength of concrete with contributions from joint shear reinforcement and $\sigma_{\mathrm{y}}$ is the column axial stress.

\section{The proposed model}

From the above analysis, it can be concluded that there is inconsistency in the research community about the contribution of strut and truss mechanisms in resisting joint shear force. The role of some parameters related to geometry, column axial load, joint shear and beam reinforcement is still under debate. In most of the joint shear strength models, the role of beam reinforcement was considered in terms of its total cross-sectional area and yield strength. Joint performance is also known to be influenced by the reinforcement-concrete bond condition, especially the bond between beam bars and the concrete at the joint core. Therefore, a new model is proposed in which the bond condition of beam bars is considered via the new parameter $\chi_{\mathrm{b}}$. The number of beam bars and their diameters are thus examined instead of their total crosssectional area and yield strength.

In the proposed model, the general form of joint shear strength is assumed to be a function of influence parameters as illustrated by

$$
\text { 7. } V_{\mathrm{jh}}=A_{\mathrm{jh}}\left(f_{\mathrm{c}}^{\prime}\right)^{0.5}\left(\sum_{i=1}^{n} a_{i} x_{i}+c\right)
$$

where $x_{i}$ are the input influence parameters, $n$ is the number of influence parameter, $a_{i}$ are the influence coefficients and $c$ is the intercept. The determination of $a_{i}$ and $c$ was based on regression analysis of the collected database.

The proposed model considers eight parameters, as shown in Table 3. Of the eight parameters, the normalised joint shear reinforcement is the sum of the normalised horizontal joint shear reinforcement and the normalised vertical joint shear reinforcement. This parameter is taken into consideration to verify whether the horizontal, the vertical or their total significantly influence joint shear strength. Table 4 illustrates the level of significance of the considered parameters on the shear strength of exterior and interior joints. The table shows the variation of the average absolute error (AAE) of the model-to-test shear strength with variation of the considered parameters. The model joint shear strength was calculated using Equation 7 with the number of influence parameters ranging from six to one. The AAE of the model to the test joint shear strength indicates the accuracy of the proposed model and was calculated using

$$
\text { 8. } \mathrm{AAE}=\frac{1}{k} \sum_{i=1}^{k} \frac{\left|V_{\mathrm{jh}, \text { model }}^{i}-V_{\mathrm{jh}, \text { test }}^{i}\right|}{V_{\mathrm{jh}, \mathrm{test}}^{i}}
$$

where $k$ is the total number of collected experimental results and $V_{\mathrm{jh}, \text { model }}^{i}$ and $V_{\mathrm{jh}, \text { test }}^{i}$ are the predicted and experimental joint shear strengths respectively.

Table 4 shows that both the horizontal joint shear reinforcement

Parameter Number of considered parameters

\begin{tabular}{lcccccccc}
\cline { 2 - 8 } & 6 & 5 & 4 & 3 & 3 & 2 & 2 & 1 \\
\hline$f_{c}^{\prime}$ & $\checkmark$ & $\checkmark$ & $\checkmark$ & $\checkmark$ & $\checkmark$ & $\checkmark$ & $\checkmark$ & $\checkmark$ \\
$\chi_{\mathrm{j}}$ & $\checkmark$ & $\checkmark$ & $\checkmark$ & $\checkmark$ & - & - & $\checkmark$ & - \\
$\chi_{\mathrm{jh}}$ & - & - & - & - & $\checkmark$ & $\checkmark$ & - & - \\
$\chi_{\mathrm{j} v}$ & - & - & - & - & $\checkmark$ & - & - & - \\
$\chi_{\mathrm{b}}$ & $\checkmark$ & $\checkmark$ & $\checkmark$ & $\checkmark$ & - & - & - & - \\
$N / b_{c} h_{\mathrm{c}} f_{\mathrm{c}}^{\prime}$ & $\checkmark$ & $\checkmark$ & $\checkmark$ & - & - & - & - & - \\
$d_{\mathrm{b}} / h_{\mathrm{c}}$ & $\checkmark$ & $\checkmark$ & - & - & - & - & - & - \\
$h_{\mathrm{c}} / h_{\mathrm{b}}$ & $\checkmark$ & - & - & - & - & - & - & - \\
$\mathrm{AAE}$, exterior joints & 0.102 & 0.105 & 0.108 & 0.143 & 0.182 & 0.191 & 0.182 & 0.213 \\
$\mathrm{AAE}$, interior joints & 0.117 & 0.117 & 0.117 & 0.122 & 0.132 & 0.155 & 0.132 & 0.162
\end{tabular}

Table 4. Variation of average absolute error of the model-to-test

joint shear strength with the considered parameters 


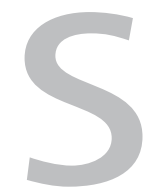

and the column intermediate longitudinal reinforcement influence the joint shear strength. The proposed new parameter, the beam bar index $\chi_{\mathrm{b}}$ significantly influences the joint shear strength. Consideration of this parameter in the model helps in reducing the AAE from $18 \cdot 2 \%$ to $14.3 \%$ for exterior joints and from $13 \cdot 2 \%$ to $12 \cdot 2 \%$ for interior joints. The two parameters with the least influence include the column depth to beam height ratio and the beam bar diameter to column depth ratio. It is worth mentioning that the beam bar index was limited to 0.4 in all the calculations, that is

$$
\chi_{\mathrm{b}}=\frac{n_{\mathrm{b}} d_{\mathrm{sb}}}{b_{\mathrm{b}}} \frac{h_{\mathrm{c}}}{h_{\mathrm{b}}} \leqslant 0 \cdot 4
$$

As the parameters $d_{\mathrm{b}} / h_{\mathrm{c}}$ and $h_{\mathrm{c}} / h_{\mathrm{b}}$ are the two least important factors, these factors were ignored to give a simple formation of the joint shear strength. Moreover, for simplicity, the influence coefficients $a_{i}$ and the intercept $c$ for exterior and interior joints were adjusted to give a unique formulation that can be applied for both types of connections. The final equation for joint shear strength is

9.

$$
V_{\mathrm{jh}}=\left(\gamma_{1}+\frac{N}{b_{\mathrm{c}} h_{\mathrm{c}} f_{\mathrm{c}}^{\prime}}+1 \cdot 2 \chi_{\mathrm{b}}\right) A_{\mathrm{jh}}\left(f_{\mathrm{c}}^{\prime}\right)^{0.5}
$$

$$
+\gamma_{2}\left(A_{\mathrm{sjh}} f_{\mathrm{jhy}}+A_{\mathrm{sjv}} f_{\mathrm{jvy}}\right)
$$

in which $\gamma_{1}=0.81$ and $\gamma_{2}=0.14$ for interior joints and $\gamma_{1}=0.34$ and $\gamma_{2}=0.22$ for exterior joints.

\section{Model verification}

The results from tests on exterior and interior joints in the compiled database were used to verify the proposed model. The test shear strength $V_{\mathrm{jh}, \text { test }}$ and the predicted shear strength $V_{\mathrm{jh} \text {,model }}$ calculated from Equation 9 are shown in Table 1 for exterior joints and Table 2 for interior joints. The tables show that the predicted joint shear strengths are in very close agreement with the experimental values. The proposed model predicts joint shear strength with an average model-to-test shear strength ratio of 1.00 and with coefficients of variation $(\mathrm{CoV})$ of $14.7 \%$ for exterior joints and $14 \cdot 0 \%$ for interior joints.

Figure 1 shows the results from the proposed model and results from the joint shear strength models proposed by ACI (2002), Kim et al. (2009), Hwang and Lee (2002) and Wang et al. (2012), corresponding to Equations 2, 4, 5 and 6 respectively. The ACI model (ACI, 2002) was chosen for the comparison because it is the simplest model and the only parameter considered is concrete compressive strength. The model of Kim et al. (2009) was chosen because it is of the same level of complexity as the proposed model and was also developed based on an empirical approach. The models of Hwang and Lee (2002) and Wang et al. (2012) were also chosen for comparison because they represent theoretical models developed based on the strut-and-tie and average stress approaches respectively. The average value of the predicted-to-test joint shear strength ratio (AVG) and its coefficient of variation $(\mathrm{CoV})$ are shown in Figure 1 to compare accuracies in predicting the joint shear strength. The figure shows that the model proposed in this paper gave the best predictions of joint shear strength.

Figures 2 and 3 show comparisons of model accuracy for exterior and interior joints respectively; the figures indicate that the proposed model can predict the shear strength of both exterior and interior connections with a great improvement in accuracy. The proposed model predicts the shear strength of exterior joints with an AAE of $11 \%$ and $\mathrm{CoV}$ of $15 \%$; these values for the most recent model (Wang et al., 2012) are $17 \%$ and $22 \%$ respectively. The ACI 352R-02 model (ACI, 2002) is the least accurate in predicting the shear strength of exterior joints, with $\mathrm{AAE}=27 \%$ and $\mathrm{CoV}=34 \%$. For interior joints (Figure 3), the proposed model predicts the joint shear strength with $\mathrm{AAE}=12 \%$ and $\mathrm{CoV}=14 \%$; the next most accurate model is ACI 352R-02, with $\mathrm{AAE}=13 \%$ and $\mathrm{CoV}=19 \%$.

\section{Parametric study}

The proposed model considers four 'key' parameters - concrete compressive strength $f_{\mathrm{c}}^{\prime}$, the normalised column axial stress $N /\left(b_{\mathrm{c}} h_{\mathrm{c}} f_{\mathrm{c}}^{\prime}\right)$, the normalised joint shear reinforcement $\chi_{\mathrm{j}}=\chi_{\mathrm{jh}}+\chi_{\mathrm{jv}}$ and the beam bar index $\chi_{\mathrm{b}}$. These parameters were double checked and compared with existing models to evaluate the fitness of the influence coefficients as well as the accuracy of the proposed model. Figure 4 illustrates the variation of the predicted-to-test joint shear strength ratio with these four parameters. Linear regression lines were added to the figure to demonstrate clearly the dependency of the joint shear strength ratio on each of the parameters. In general, the figure shows that the proposed joint shear strength ratio has no clear dependence on these four parameters despite their large range variation. Figure 4 also reveals that the proposed model gives the predictedto-test joint shear strength ratio with less scatter than the other models. In particular, the figure shows that the predicted-to-test joint shear strength ratio of the proposed model varies around 1.00 in a small range from 0.71 to 1.51 ; this range for the models of ACI (2002), Kim et al. (2009) and Wang et al. (2012) is 0.57$1 \cdot 77,0 \cdot 61-1.83$ and $0 \cdot 51-1.66$ respectively.

Figure 4(a) compares the dependence of joint shear strength ratio on concrete compressive strength for the proposed model and the model in ACI 352R-02. In both models, the power term for concrete compressive strength is 0.5 . However, the joint shear strength ratio of the ACI model tends to reduce with an increase in concrete strength while there is no clear dependency in the proposed model. This indicates that consideration of the remaining three parameters effectively improves the reliability of the proposed model.

Figure 4(b) compares the variation of joint shear strength ratio 
A new empirical model for shear strength of reinforced concrete beam-column connections

Tran, Hadi and Pham

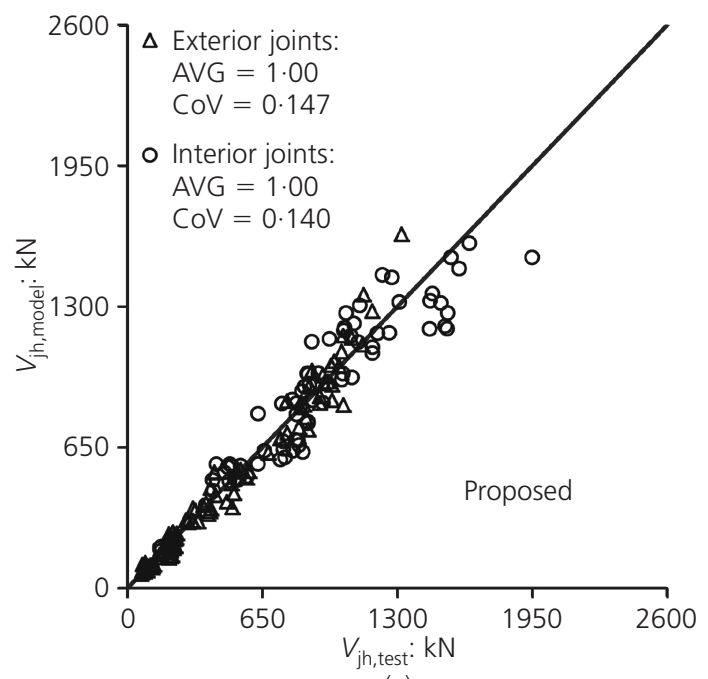

(a)

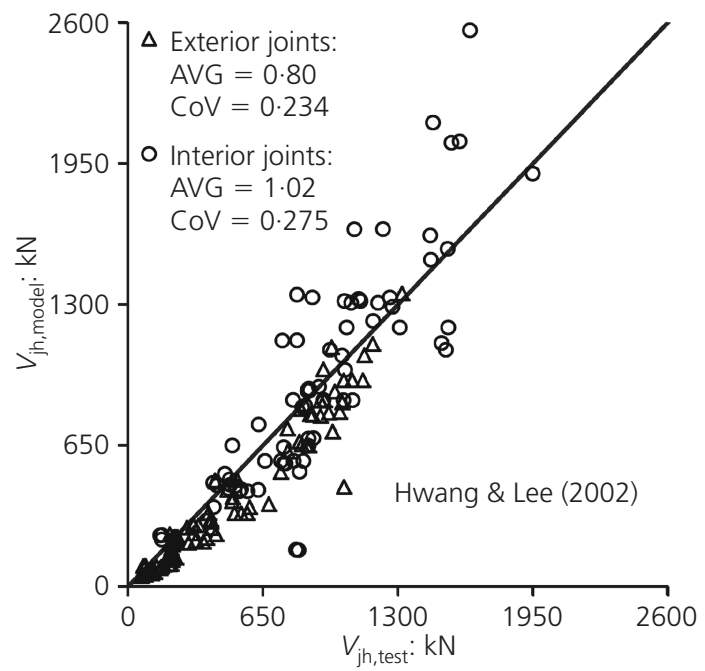

(c)

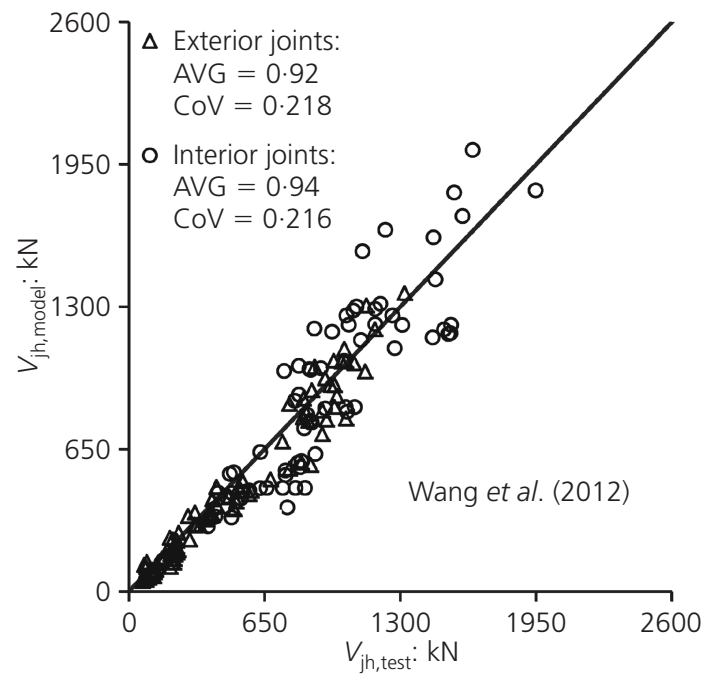

(e)

Figure 1. Performance of the proposed model (a) and the models of $\mathrm{ACl}$ (2002) (b), Hwang and Lee (2002) (c), Kim et al. (2009) (d) and Wang et al. (2012) (e)

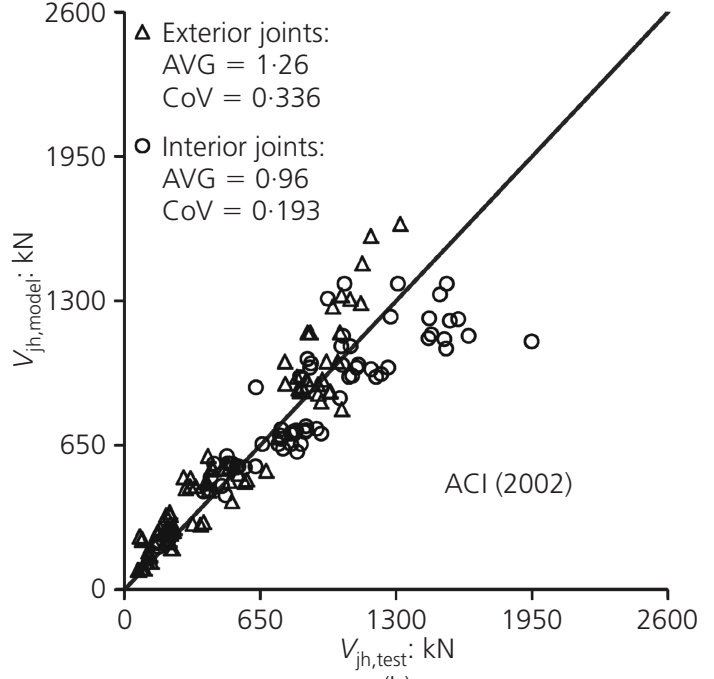

(b)

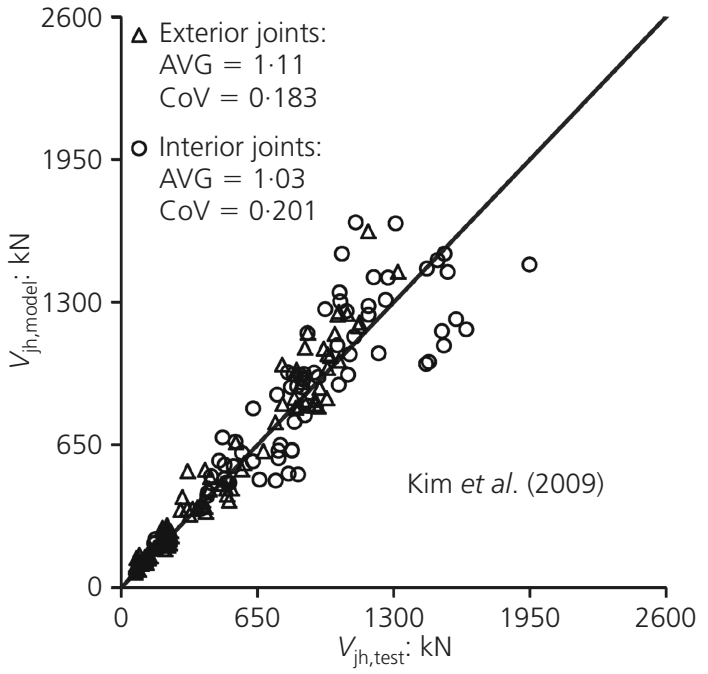

(d) 


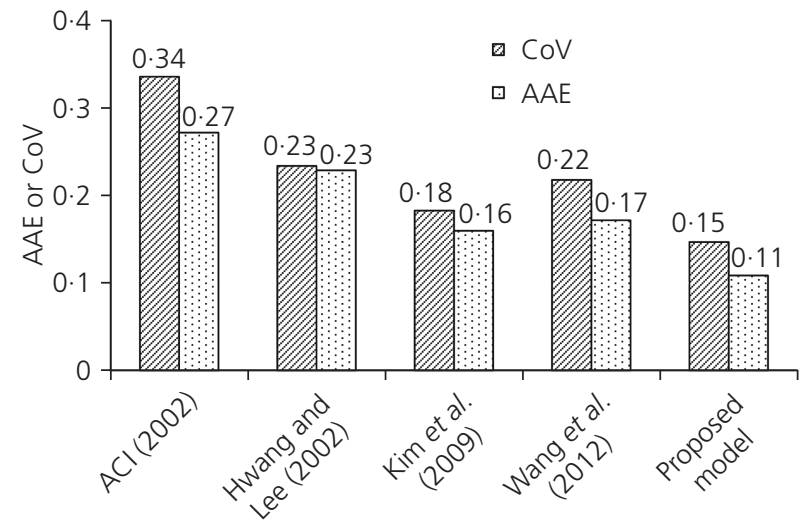

Figure 2. Accuracy comparison of the models for shear strength prediction of exterior joints

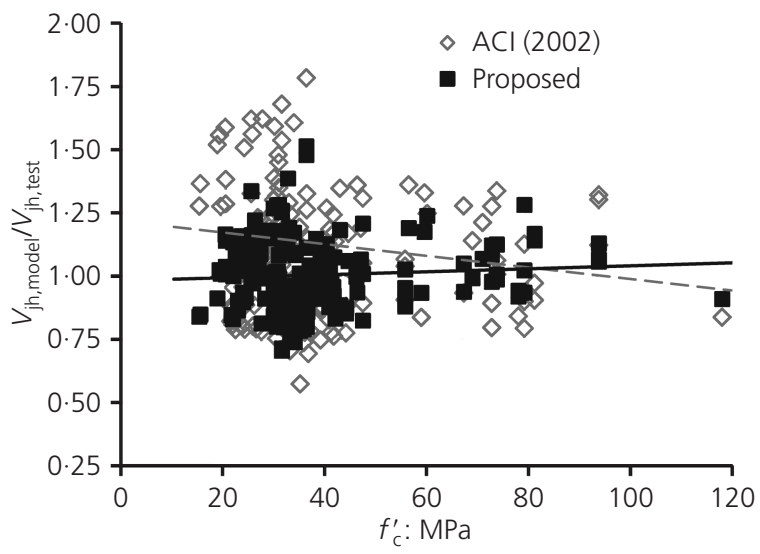

(a)

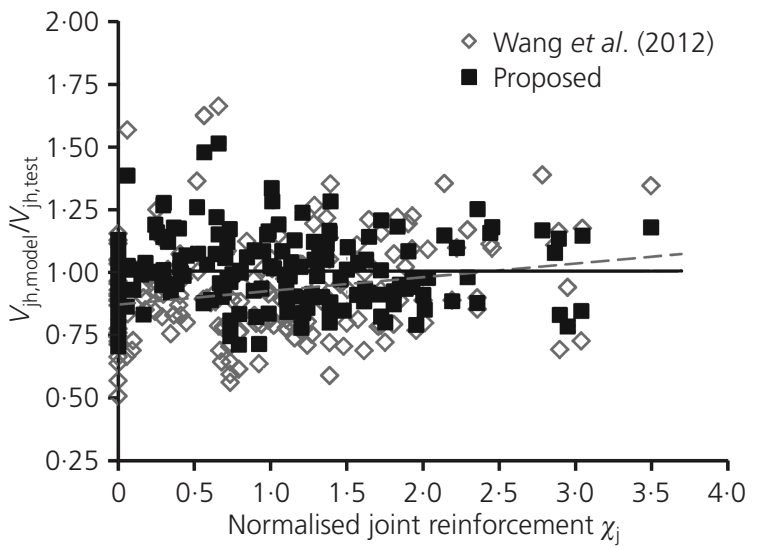

(c)

Figure 4. Variation of joint shear strength ratio with each of the influence parameters

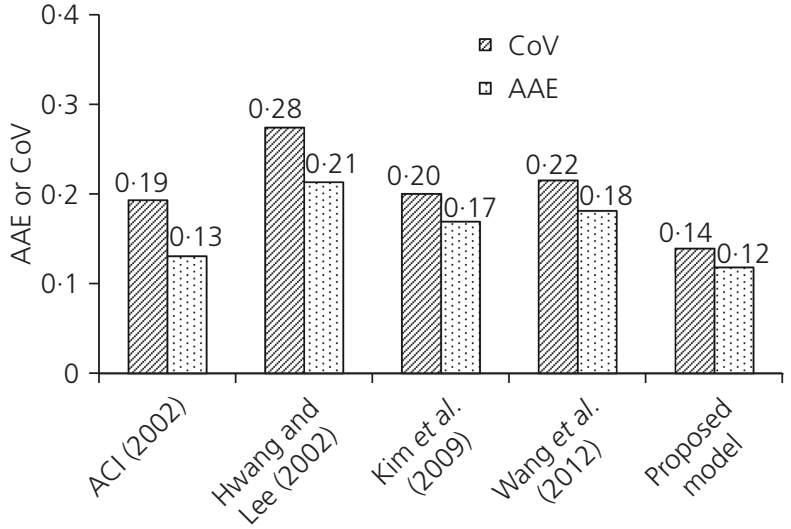

Figure 3. Accuracy comparison of the models for shear strength prediction of interior joints

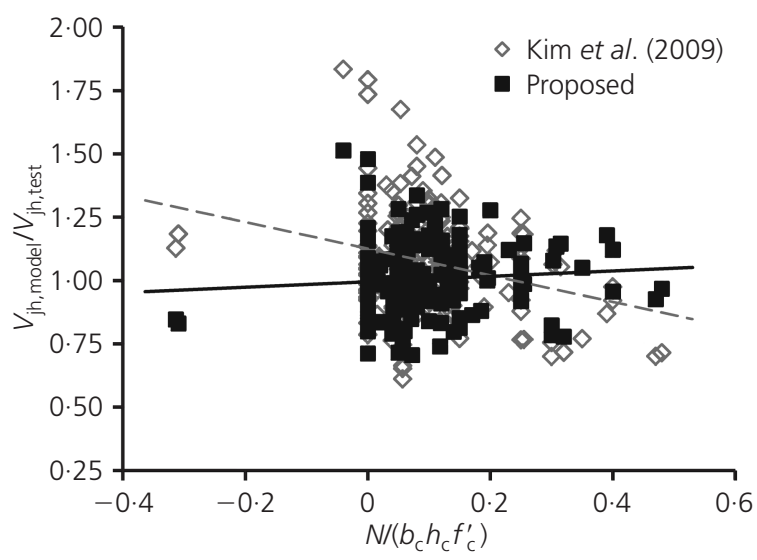

(b)

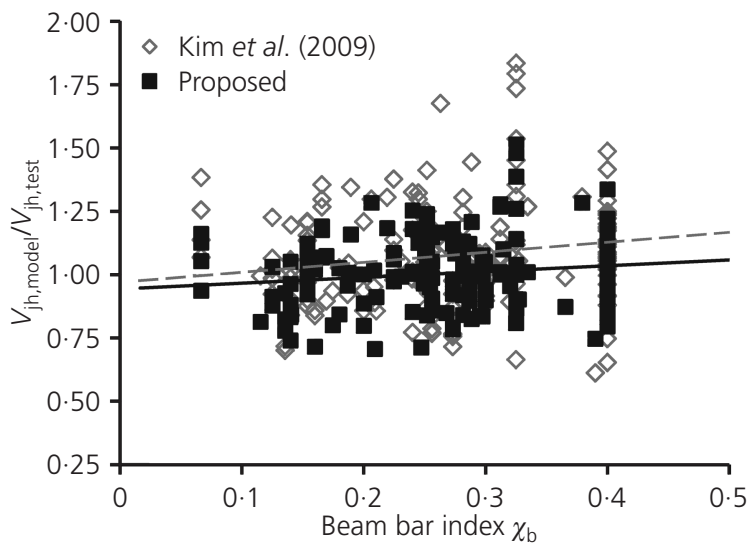

(d) with the normalised column axial stress for the proposed model and the model of Kim et al. (2009). Both models were developed based on an empirical approach, but the model of Kim et al. neglects the influence of column axial load and thus its joint shear strength ratio is significantly influenced by the normalised column axial stress.

The dependency of the joint shear strength ratio on the normalised 
joint shear reinforcement in the proposed model and the model of Wang et al. (2012) is shown in Figure 4(c). Once again, it can be seen that the proposed model has the better performance. The independence of the proposed joint shear strength ratio on the normalised joint shear reinforcement indicates that the proposed coefficient $\gamma_{2}$ in Equation 9 is justified.

Figure 4(d) shows a comparison of the dependence of joint shear strength ratio on the beam bar index. For the proposed model, there is very little dependency of the joint shear strength ratio on the beam bar index, while significant dependency is observed for the model of Kim et al. (2009). Moreover, as there was no limitation in the variation range of the amount of beam steel bars, it is believed that the model of Kim et al. overestimates the joint shear strength when the amount of beam reinforcement is high. It is noted that, in both models, the role of the beam steel bars was considered in different manners: Kim et al. considered the beam bars in terms of their total area together with their yield strength while the proposed model considers their contact area with the surrounding concrete. The better performance of the proposed model compared with that of Kim et al. in this respect indicates that the reasons for proposing the parameter $\chi_{\mathrm{b}}$ to account for the influence of beam longitudinal reinforcement are rational.

\section{Conclusions}

A new empirical model for joint shear strength of RC beamcolumn connections has been introduced. The model was developed based on regression analysis using a large database collected from the literature. The influence of some 'key' parameters on joint shear strength was analysed and four parameters were chosen to generate the proposed model. These four parameters included a new parameter to reflect the beam bar bond condition as well as the possibility of the beam bar transferring a fraction of the joint shear force into the column. The regression analysis showed that consideration of this parameter helps to significantly improve the reliability of the model when compared with test results.

A parametric study to illustrate the better performance of the proposed model when compared with other models was also conducted. This showed that the proposed coefficients in the equation for joint shear strength are justified and that the proposed equation for joint shear strength is reliable despite the large variation in the parameters of influence. Results from the proposed model were compared with those from four existing joint shear strength models to demonstrate the effectiveness of the new model. Due to its accuracy, stability and simplicity, application of the proposed model for predicting the shear strength of beam-column connections in practical design is expected.

\section{REFERENCES}

$\mathrm{ACl}$ (American Concrete Institute) (2002) ACI 352R-02:

Recommendations for design of beam-column joints in monolithic reinforced concrete structures. ACI, Farmington Hills, MI, USA.
ACI (American Concrete Institute) (2008) ACI 318R: Building code requirements for structural concrete and commentary. ACI, Farmington Hills, MI, USA.

AlJ (Architectural Institute of Japan) (1999) Design guideline for earthquake resistance reinforced concrete buildings based on inelastic displacement concept. AIJ, Tokyo, Japan.

Alva GMS, Debs ALHDC and Debs MKE (2007) An experimental study on cyclic behaviour of reinforced concrete connections. Canadian Journal of Civil Engineering 34(4): 565-565.

Antonopoulos CP and Triantafillou TC (2003) Experimental investigation of FRP-strengthened RC beam-column joints. Journal of Composites for Construction 7(1): 39-49.

Attaalla SA (2004) General analytical model for nominal shear stress of type 2 normal and high-strength concrete beamcolumn joints. ACI Structural Journal 101(6): 881-882.

Au FTK, Huang K and Pam HJ (2005) Diagonally-reinforced beam-column joints reinforced under cyclic loading. Proceedings of the Institution of Civil Engineers - Structures and Buildings 158(1): 21-40.

Bakir PG and Boduroğlu HM (2002) A new design equation for predicting the joint shear strength of monotonically loaded exterior beam-column joints. Engineering Structures 24(8): 1105-1117.

BSI (2004) Eurocode 8: Design of structures for earthquake resistance. Part 1: General rules, seismic actions and rules for buildings. BSI, London, UK.

Chalioris CE, Favvata MJ and Karayannis CG (2008) Reinforced concrete beam-column joints with crossed inclined bars under cyclic deformations. Earthquake Engineering and Structural Dynamics 37(6): 881-897.

Choi H and Kim J (2011) Progressive collapse-resisting capacity of RC beam-column sub-assemblage. Magazine of Concrete Research 63(4): 297-310.

Chun SC, Oh B, Lee SH and Naito CJ (2009) Anchorage strength and behavior of headed bars in exterior beam-column joints. ACI Structural Journal 106(5): 579-590.

Chutarat N and Aboutaha RS (2003) Cyclic response of exterior reinforced concrete beam-column joints reinforced with headed bars-experimental investigation. ACI Structural Journal 100(2): 259-264.

Clyde C, Pantelides CP and Reaveley LD (2000) Performance-based evaluation of reinforced concrete building exterior joints for seismic excitation. Earthquake Spectra 18(3): 449-480.

Dhakal RP, Pan TC, Irawan P et al. (2005) Experimental study on the dynamic response of gravity-designed reinforced concrete connections. Engineering Structures 27(1): 75-87.

Durrani A and Wight J (1985) Behaviour of interior beam-tocolumn connections under earthquake-type loading. $A C I$ Journal 82(3): 343-349.

Ehsani MR and Alameddine F (1991) Design recommendations for type 2 high-strength reinforced concrete connections. ACI Structural Journal 88(3): 277-291.

Ehsani MR and Wight JK (1985) Exterior reinforced concrete beam-to-column connections subjected to earthquake-type loading. ACI Structural Journal 82(4): 492-499. 
Ehsani MR, Moussa AE and Vallenilla CR (1987) Comparison of inelastic behavior of reinforced ordinary and high-strength concrete frames. ACI Structural Journal 84(2): 161-169.

El-Amoury T and Ghobarah A (2002) Seismic rehabilitation of beam-column joint using GFRP sheets. Engineering Structures 24(11): 1397-1407.

Fisher MJ and Sezen H (2011) Behavior of exterior reinforced concrete beam-column joints including a new reinforcement. Structural Engineering and Mechanics 40(6): 867-883.

Ghobarah A and El-Amoury T (2005) Seismic rehabilitation of deficient exterior concrete frame joints. Journal of Composites for Construction 9(5): 408-408.

Ghobarah A and Said A (2001) Seismic rehabilitation of beamcolumn joints using FRP laminates. Earthquake Engineering 5(1): 113-129.

Ghobarah A and Said A (2002) Shear strengthening of beamcolumn joints. Engineering Structures 24(7): 881-888.

Hanson NW and Connor HW (1967) Seismic resistance of reinforced concrete beam-column joints. Journal of Structural Division ASCE 93(5): 533-559.

Hegger J, Sherif A and Roeser W (2003) Nonseismic design of beam-column joints. ACI Structural Journal 100(5): 654664.

Hwang J and Lee HJ (1999) Analytical model for predicting shear strengths of exterior reinforced concrete beam-column joints for seismic resistance. ACI Structural Journal 96(5): 846857.

Hwang SJ and Lee HJ (2002) Strength prediction for discontinuity regions by softened strut-and-tie model. Journal of Structural Engineering ASCE 128(12): 1519-1526.

Hwang SJ, Lee HJ and Wang KC (2004) Seismic design and detailing of exterior reinforced concrete beam-column joints. Proceedings of 13th World Conference on Earthquake Engineering, Vancouver BC, Canada, paper 0397.

Hwang SJ, Lee HJ, Liao TF, Wang KC and Tsai HH (2005) Role of hoops on shear strength of reinforced concrete beam-column joints. ACI Structural Journal 102(3): 445-453.

Ishibashi K (1993) Experimental Study on Interior Beam-tocolumn Joints of Reinforced Concrete Frames. ??? thesis Tokyo University, Tokyo, Japan (in Japanese).

Joyklad P, Pimanmas A and Dhakal RP (2012) Cyclic performance of beam-column joints with extended column fixed at base. Part I: experimental investigation. Magazine of Concrete Research 64(9): 807-807.

Kaku T and Asakusa H (1991) Ductility estimation of exterior beam-column subassemblages in reinforced concrete frames. On-line Journals Search Results 123(xxx): 167-186.

Karayannis CG and Sirkelis GM (2005) Response of column and joints with spiral shear reinforcement. Proceedings of CMEM Conference on Computional Methods and Experimental Measurements, Xxxxxuxxxxxxx, Malta.

Karayannis CG and Sirkelis GM (2008) Strengthening and rehabilitation of RC beam-column joints using carbon-FRP jacketing and epoxy resin injection. Earthquake Engineering and Structural Dynamics 37(5): 769-790.
Karayannis CG, Chalioris CE and Sirkelis GM (2008) Local retrofit of exterior RC beam-column joints using thin RC jackets an experimental study. Earthquake Engineering and Structural Dynamics 37(5): 727-746.

Kim J and LaFave JM (2007) Key influence parameters for the joint shear behaviour of reinforced concrete (RC) beamcolumn connections. Engineering Structures 29(10): 2523 2539.

Kim J and LaFave JM (2008) Probabilistic joint shear strength models for design of RC beam-column connections. ACI Structural Journal 105(6): 770-780.

Kim J and Yu J (2012) Analysis of reinforced concrete frames subjected to column loss. Magazine of Concrete Research 64(1): $21-33$.

Kim J, LaFave JM and Song J (2009) Joint shear behaviour of reinforced concrete beam-column connections. Magazine of Concrete Research 61(2): 119-132.

Kitayama K, Otani S and Aoyama H (1991) Development of design criteria for RC interior beam-column joints. Design of Beam-Column Joints for Seismic Resistance. American Concrete Institute, Detroit, MI, USA, ACI SP-123, pp. $97-$ 123.

Kuang JS and Wong HF (2006) Effects of beam bar anchorage on beam-column joint behaviour. Proceedings of the Institution of Civil Engineers - Structures and Buildings 159(2): 115124.

Le-Trung K, Lee J, Lee DH, Lee K and Woo S (2010) Experimental study of RC beam-column joints strengthened using CFRP composites. Composites Part B 41(1): 76-85.

Lee WT, Chiou YJ and Shih MH (2010) Reinforced concrete beam-column joint strengthened with carbon fiber reinforced polymer. Composite Structures 92(1): 48-60.

Leon RT (1990) Shear-strength and hysteretic behavior of interior beam-column joints. ACI Structural Journal 87(1): 3-11.

Lima C, Martinelli E and Faella C (2012) Capacity models for shear strength of exterior joints in RC frames: state-of-the-art and synoptic examination. Bulletin of Earthquake Engineering 10(3): 967-983.

Liu C (2006) Seismic Behaviour of Beam-Column Joint Subassemblies Reinforced with Steel Fibres. Masters thesis, University of Canterbury, Christchurch, New Zealand.

Lu X, Urukap TH, Li S and Lin F (2012) Seismic behavior of interior RC beam-column joints with additional bars under cyclic loading. Earthquakes and Structures 3(1): 37-57.

Marques JLG and Jirsa JO (1975) A study of hooked bar anchorages in beam-column joints. ACI Structural Journal 72(5): 198-209.

Megget LM (1974) Cyclic behaviour of exterior reinforced concrete beam-column joints. Bulletin of The New Zealand National Society for Earthquake Engineering 7(1): 27-47.

Meinheit DF and Jirsa JO (1977) The Shear Strength of Reinforced Concrete Beam-Column Joints. University of Texas, Austin, TX, USA, CESRL Report 77-1.

Morita S, Kitayama K, Koyama A and Hosono T (1999) Effects of beam bar bond and column axial load on shear strength in 
reinforced concrete interior beam-column joints. Transactions of the Japan Concrete Institute 21(xxx): 453-460.

Murty CVR, Rai DC, Bajpai KK and Jain SK (2003) Effectiveness of reinforcement details in exterior reinforced concrete beam-column joints for earthquake resistance. ACI Structural Journal 100(2): 149-156.

Najafian HA, Vollum RL and Fang L (2013) Comparative assessment of finite-element and strut and tie based design methods for deep beams. Magazine of Concrete Research 65(16): 970-970.

Noguchi H and Kashiwazaki T (1992) Experimental studies on shear performances of RC interior column-beam joints with high-strength materials. Proceedings of 10th World Conference on Earthquake Engineering, Zxxmxxxxxxx, Xxxxxxxyxaxxx.

Noguchi H and Kurusu K (1988) Correlation of bond and shear in $\mathrm{RC}$ beam-column connections subjected to seismic forces. Proceeding of 9th World Conference on Earthquake Engineering, Xxxxxxxxxxxx, Xxxxxxxxxxxxxx, pp. 597-602. Oka K and Shiohara H (1992) Test of high-strength concrete interior beam-to-column joint subassemblages. Proceedings of 10th World Conference on Earthquake Engineering, Хxхxхxхxхxхxx, Хxхxхххххххxхxх, pp. 3211-3217.

Otani S, Kobayashi Y and Aoyama H (1984) Reinforced concrete beam-column joints under simulated earthquake loading. Proceedings of 1st US-NZ-Japan Seminar-Monterey.

Pantazopoulou S and Bonacci J (1992) Consideration of questions about beam-column joints. ACI Structural Journal 89(1): $27-36$.

Pantelides CP, Hansen J, Nadauld J and Reaveley LD (2002) Assessment of RC Building Exterior Joints with Substandard Details. University of Utah, Publisher location, PEER Report 2002/18.

Park R and Paulay T (1974) Behaviour of reinforced concrete external beam-column joints under cyclic loading. Proceedings of $5 \mathrm{~h}$ World Conference on Earthquake Engineering, Rome, Italy.

Park S and Mosalam KM (2012) Parameters for shear strength prediction of exterior beam-column joints without transverse reinforcement. Engineering Structures 36(xxx): 198-209.

Parker DE and Bullman PJM (1997) Shear strength within reinforced concrete beam-column joints. Structural Engineer 75(4): $53-57$.

Patel PA, Desai AK and Desai JA (2013) Evaluation of RC and SFRC exterior beam-column joint under cyclic loading for reduction in lateral reinforcement of the joint region. Magazine of Concrete Research 65(7): 405-414.

Paulay T and Priestley MJN (1992) Seismic Design of Reinforced Concrete and Masonry Buildings. Wiley, New York, NY, USA.

SANZ (Standards Association of New Zealand) (1995) NZS-3101: Concrete structures standard. Part 1: the design of concrete structures. SANZ, Wellington, New Zealand.

Sarsam KF and Phipps ME (1985) The shear design of in situ reinforced concrete beam-column joints subjected to monotonic loading. Magazine of Concrete Research 37(130): $16-28$.

Shrestha R, Smith ST and Samali B (2009) Strengthening RC beam-column connections with FRP strips. Proceedings of the Institution of Civil Engineers - Structures and Buildings 162(5): 323-334.

Supaviriyakit T and Pimanmas A (2008) Comparative performance of sub-standard interior reinforced concrete beam-column connection with various joint reinforcing details. Materials and Structures 41(3): 543-557.

Tsonos AG (2007) Cyclic load behavior of reinforced concrete beam-column subassemblages of modern structures. $A C I$ Structural Journal 104(4): 468-478.

Tsonos AG, Tegos IA and Penelis GG (1992) Seismic resistance of type 2 exterior beam-column joints reinforced with inclined bars. ACI Structural Journal 89(1): 3-12.

Vatani-Oskouei A (2010) Repairing of seismically damaged RC exterior beam-column connection using CFRP. Journal of Reinforced Plastics and Composites 29(21): 3257-3274.

Vollum RL and Newman JB (1999) Strut and tie models for analysis/design of external beam-column joints. Magazine of Concrete Research 51(6): 415-425.

Wang GL, Dai JG and Teng JG (2012) Shear strength model for RC beam-column joints under seismic loading. Engineering Structures 40(xxx): $350-360$.

Wang YC and Hsu K (2009) Shear strength of RC jacketed interior beam-column joints without horizontal shear reinforcement. ACI Structural Journal 106(2): 222-232.

Wong HF and Kuang JS (2008) Effects of beam-column depth ratio on joint seismic behaviour. Proceeding of the Institution of Civil Engineers - Structures and Buildings 161(2): 91101.

Zhang L and Jirsa JO (1982) A Study of Shear Behavior of Reinforced Concrete Beam-Column Joints. University of Texas, Austin, TX, USA, PMFSEL Report 82-1.

\section{WHAT DO YOU THINK?}

To discuss this paper, please submit up to 500 words to the editor at journals@ice.org.uk. Your contribution will be forwarded to the author(s) for a reply and, if considered appropriate by the editorial panel, will be published as a discussion in a future issue of the journal. 
1: Sarsam and Phillips (1985) is not in reference list. Please give full details of this work or advise changes to citation

2: Notation lists $\alpha$ as inclination of diagonal strut: please clarify

3: We have $h_{\mathrm{c}} / h_{\mathrm{b}}$ in Table 3, not $h_{\mathrm{b}} / h_{\mathrm{c}}:$ OK?

4: What is $d_{\mathrm{b}}$ ?? Please add to notation

5: What type of thesis? PhD? MSc

6: Please give journal title

7: Please provide issue nu

8: Please give town/city

10: Please provide issue number

if appicable and country) of conference

11: Please give location (town/cins state if applicable and country) of conference

12: If published, please give name and location of publisher and page numbers of art

13: Please give location (town/city, US state if applicable and country) of conference

14: Please give name and location (town/city, US state if applicable and country) of

15: Please give location (town/city, US state if applicable and country) of conference

16: Please give name and location (town/city, US state if applicable and country) of

17: Please give more details: Monterey where?

18: If published, please give name and location ublisher and page numbers of article

19: Please provide publisher location $\bigcirc$

20: If published, please give name an ation of publisher and page numbers of article

21: Please provide issue number<smiles>C1=Cc2cccc1c2</smiles>

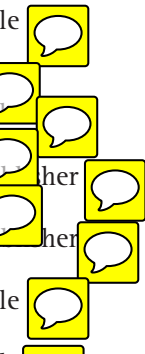

